\title{
Phenomenological models for transient NAPL-water mass-transfer processes
}

\author{
Susan E. Powers ${ }^{a}$, Linda M. Abriola ${ }^{b}$, Joyce S. Dunkin ${ }^{c}$, \\ Walter J. Weber, Jr. ${ }^{b}$ \\ ${ }^{a}$ Department of Civil and Environmental Engineering. Clarkson University, Potsdam, NY 13699-5715, USA \\ 'Department of Civil and Environmental Engineering, The University of Michigun, Ann Arbor. \\ MI 48109-2125, USA \\ 'Limno-Tech, Inc., 2395 Huron Parkway, Ann Arbor, MI 48104. USA
}

(Received October 8, 1993; revision accepted January 26, 1994)

\begin{abstract}
A phenomenological model for transient NAPL dissolution, referred to as the "sphere" model, is developed. In this model, the mass-transfer coefficient and specific surface areas are quantified independently. The unique aspect of this work is the assumption that the complex distribution of NAPL blob shapes and sizes can be represented by a set of spheres with a range of diameters. The sphere diameter distributions are determined through the entrapment, polymerization and characterization of styrene blobs in sandy media. Mass-transfer coefficients are estimated from experimental measurements of the dissolution of naphthalene emplaced as solid spheres within sandy media.

The phenomenological model describing NAPL dissolution rates is incorporated into a solute transport equation and solved with a numerical simulator. Simulations show that the sphere model can be calibrated to experimental NAPL dissolution data from column studies presented previously. The inclusion of a range of sphere sizes resulted in a model which represents experimental data better than a model based on mono-size spheres. The sphere model also adequately predicts dissolution for NAPL's with a range of solubilitics provided the NAPL blob size distributions can be adequately estimated.

The primary limitation of the sphere model is the quantity of input data required to describe the distribution of effective sphere sizes. While these data requirements are extensive, the ability to define the NAPL blob distribution within the model makes the sphere model much more versatile for extrapolation to heterogeneous systems than other available dissolution models.
\end{abstract}




\section{Notation}

List of symbols used in this paper

\begin{tabular}{lll}
\hline Symbol & Description & Dimension \\
\hline$a_{o}$ & specific surface area & {$\left[\mathrm{L}^{-1}\right]$} \\
$C_{s}$ & equilibrium concentration & {$\left[\mathrm{M} \mathrm{L}^{-3}\right]$} \\
$C$ & aqueous-phase concentration & {$\left[\mathrm{M} \mathrm{L}^{-3}\right]$} \\
$d$ & diameter & {$[\mathrm{L}]$} \\
$D_{h}$ & hydrodynamic dispersion coefficient & {$\left[\mathrm{L}^{2} \mathrm{~T}^{-1}\right]$} \\
$D_{L}$ & free liquid diffusivity in water & {$\left[\mathrm{L}^{2} \mathrm{~T}^{-1}\right]$} \\
$f_{r}$ & mass fraction retained on a sieve & $(-)$ \\
$F$ & calibration parameter & $(-)$ \\
$k_{f}$ & mass-transfer coefficient & {$\left[\mathrm{L} \mathrm{T}^{-1}\right]$} \\
$\hat{k}$ & lumped mass-transfer coefficient & {$\left[\mathrm{T}^{-1}\right]$} \\
$L$ & length of column & {$[\mathrm{L}]$} \\
$l_{c}$ & characteristic length & {$[\mathrm{L}]$} \\
$m$ & mass & {$[\mathrm{M}]$} \\
$N$ & number & $(-)$ \\
$q$ & superficial aqueous-phase velocity & {$\left[\mathrm{L} \mathrm{T}^{-1}\right]$} \\
$S$ & phase saturation & $(-)$ \\
$S$ & source of solute & {$\left[\mathrm{M} \mathrm{T}^{-1} \mathrm{~L}^{-3}\right]$} \\
$U_{1}$ & iniformity index & $(-)$ \\
$V$ & volume & {$\left[\mathrm{L}^{3}\right]$} \\
$x$ & distance & {$[\mathrm{L}]$} \\
$\beta_{1}$ & correlation exponent for Re & $(-)$ \\
$\epsilon$ & porosity & $(-)$ \\
$\delta$ & normalized diameter & $(-)$ \\
$\mu$ & viscosity & {$\left[\mathrm{M} \mathrm{L}^{-1} \mathbf{T}^{-1}\right]$} \\
$\rho$ & density & {$\left[\mathrm{M} \mathrm{L} \mathrm{L}^{-3}\right]$} \\
$\theta$ & volumetric fraction & $(-)$ \\
& &
\end{tabular}

Subscripts:

$\begin{array}{ll}\text { b } & \text { blob } \\ \text { eq } & \text { equilibrium } \\ \text { g } & \text { sand grain } \\ \text { i } & \text { organic species } \\ \text { n } & \text { NAPL (nonaqueous-phase liquid) } \\ \text { s } & \text { sphere } \\ \text { w } & \text { water } \\ 50 & \text { median }\end{array}$

Dimensionless variables:

$\begin{array}{ll}\mathrm{Pe} & \text { Péclet number }\left(\mathrm{Pe}=q L / D_{L}\right) \\ \mathrm{Re} & \text { Reynolds number }\left(\operatorname{Re}=q \rho_{w_{w}} l_{c} / \mu_{w}\right) \\ \operatorname{Re}^{\prime} & \operatorname{Reynolds~number~}\left(\operatorname{Re}^{\prime}=q \rho_{w} l_{c} /\left[\mu_{w^{\prime}}\left(\epsilon-\theta_{n}\right)\right)\right) \\ \mathrm{Sc} & \text { Schmidt number }\left(\mathrm{Sc}=\mu_{w} / D_{L} \rho_{w}\right) \\ \mathrm{Sh} & \text { Sherwood number }\left(\mathrm{Sh}=k_{f} l_{c} / D_{L}\right) \\ \mathrm{Sh}^{\prime} & \text { Sherwood number }\left(\mathrm{Sh}^{\prime}=\hat{k} d_{s 0}^{2} / D_{L}\right) \\ \chi & \text { normalized concentration }\left(\chi=C / C_{s}\right)\end{array}$


Notation (continued)

$\begin{array}{ll}\xi & \text { dimensionless distance }(\xi=x / L) \\ \tau & \text { dimensionless time }(\tau=q 1 / L) \\ \alpha & \text { dimensionless specific surface area }\left(\alpha=a_{o} L\right)\end{array}$

\section{Introduction}

Many of U.S.A.'s most persistent groundwater contamination problems involve the presence of nonaqueous-phase liquids (NAPL's) (Feenstra and Coburn, 1986; Mackay and Cherry, 1989; Mercer and Cohen, 1990). The use of pump-and-treat remediation techniques at such NAPL-contaminated sites has not been proven an effective means of removing entrapped organic liquid, which functions as a source of contamination to flowing groundwater (Keely, 1989). Over time, the rate of removal of the organic contaminants tends to decrease substantially, and concentrations of contaminants in the groundwater are often observed to rise after pumping has ceased (Cherry et al., 1990). One explanation for these observations is rate-limited exchange of mass between the entrapped organic liquid and aqueous phases (Abriola, 1989).

The potential significance of mass-transfer rate limitations on the efficiency of groundwater remediation in the presence of NAPL's has been explored by a number of researchers (Hunt et al., 1988; Powers et al., 1991; Borden and Kao, 1992; Johnson and Pankow, 1992). Powers et al. (1991) conducted a theoretical assessment of NAPL dissolution rates and showed that, over the period of time required for remediation, concentrations of an organic species downgradient of a NAPL spill site would tend to decrease as the volume of NAPL is reduced. Such behavior would result in aquifer remediation times significantly greater than those predicted by equilibrium considerations. The potential importance of rate-limited NAPL dissolution is also supported by a number of laboratory column investigations (e.g., Powers et al., 1992, 1994; Geller and Hunt, 1993; Imhoff et al., 1994). These studies show that, at the laboratory-scale, the interphase exchange of soluble species is a rate-limited process for coarse or graded media at high aqueous-phase velocities, or after extended periods of time when the interfacial area is substantially reduced. Studies of larger-scale systems have shown that the pools of NAPL which accumulate on top of capillary barriers also result in rate-limited NAPL dissolution (Anderson et al., 1992).

Innovative technologies for aquifer remediation such as surfactant flushing and soil venting also rely on interphase mass-transfer mechanisms. Limited laboratory investigations of these processes suggest that rate-limited mass transfer will occur under many conditions. In surfactant flushing experiments, effluent concentrations from columns containing a pool of NAPL (Fountain et al., 1991) or residual NAPL blobs (Pennell et al., 1993) have been observed at concentrations much lower than the equilibrium concentration of the organic species. Pennell et al. (1993) report that mass-transfer coefficients determined for surfactant 
flushing experiments are significantly less than those observed in water-NAPL system.s.

Due to the potential significance of mass-transfer rate limitations for processes designed to reduce entrapped NAPL saturations in the subsurface, phenomenological models quantifying these rates will be required to permit adequate evaluation of these technologies. In this paper, a phenomenological model for NAPL-water interphase mass transfer is developed and applied to data from one-dimensional NAPL dissolution column experiments. This model is based on a linear-drivingforce mass-transfer relationship, and utilizes independent measures of mass-transfer coefficients and the NAPL-water interfacial area.

\section{Background}

\subsection{NAPL migration and entrapment}

Migration of NAPL's in subsurface systems is a complex process. Upon introduction to the subsurface, NAPL's tend to migrate downward through the vadose zone due to gravitational forces. If the NAPL is less dense than water (e.g., most petroleum hydrocarbons), it will spread laterally in the capillary fringe upon reaching the water table, creating a lens of high NAPL saturation (van Dam, 1967). Such light NAPL's may subsequently become entrapped in the saturated zone due to water-table fluctuations. In contrast, NAPL's which are heavier than water (e.g., mos! chlorinated solvents), will spread laterally through the capillary fringe until the entry pressure of the water-saturated medium is overcome (Cohen et al., 1993). With sufficient head, the NAPL will enter and migrate downward into the saturated zone (van Dam, 1967; Schwille, 1988). Interfacial forces acting between the water phase and the NAPL will cause "blobs" or "ganglia" of the organic phase to be retained within the saturated zone. Entrapment of the organic phase as discrete blobs is controlled by a complex interaction of soil and fluid characteristics.

The entrapment process and resulting general shape of NAPL blobs will be controlled by capillary phenomena. Entrapped blobs of a nonwetting fluid, typically the NAPL, have concave interfaces and are formed within a single pore or several adjacent pore bodies (Schwille, 1988). Accordingly, the nonwetting phase enters large pores preferentially during natural gravitational drainage. Blobs can become detached from the continuous organic fluid phase when capillary forces are sufficiently strong to overcome viscous and gravity or buoyancy forces (Wardlaw, 1982; Mohanty et al., 1987).

The dimensionless capillary and Bond numbers describe the relative magnitudes of these forces (Morrow and Chatzis, 1981). The capillary number (Ca) is typically defined as (see the Notation for symbols used in this paper):

$$
\mathrm{Ca}=\mu_{\mathrm{w}} q / \sigma_{\mathrm{nw}}
$$


where $\mu_{\mathrm{w}}$ is the viscosity of the aqueous phase; $q$ is the superficial velocity; and $\sigma_{\mathrm{nw}}$ is the interfacial tension between the aqueous and organic phases. This dimensionless number relates viscous forces associated with groundwater flow to interfacial forces between the aqueous-phase and NAPL blobs. During waterflooding at low fluid velocities $\left(\mathrm{Ca} \leqslant 2 \cdot 10^{-5}\right)$, capillary forces dominate viscous forces. In this range, there is a weak inverse relationship between blob size and capillary number (Mayer and Miller, 1993). As the capillary number is increased, $\mathrm{Ca}>2 \cdot 10^{-5}$, viscous forces become significant and the volume of entrapped NAPL decreases $(\mathrm{Ng}$ et al., 1978). The Bond number $(\mathrm{Bn})$ is a ratio of buoyancy forces to capillary forces:

$$
\mathrm{Bn}=\Delta \rho g R^{2} / \sigma_{\mathrm{nw}}
$$

where $\Delta \rho$ is the density difference between the fluids; $g$ is the acceleration due to gravity; and $R$ is a representative pore dimension, frequently taken as the grain diameter. The size of entrapped blobs decreases with increasing Bond number (Mayer and Miller, 1993). That is, smaller blobs, resulting in larger specific interfacial areas, are expected as grain sizes or density differences decrease, or as interfacial tension increases.

Under conditions of relatively constant capillary and Bond numbers, the pore geometry is a critical parameter defining the distribution of residual NAPL blob sizes. In a soil with a high pore-body-to-throat size ratio (pore aspect ratio), NAPL tends to "snap-off" in individual pore bodies (Chatzis et al., 1983; Mohanty et al., 1987; Morrow et al., 1988). Singlet blobs, which exist within one pore body and are formed by the snap-off mechanism, are the predominant type of blob in well-sorted unconsolidated sands (Larson et al., 1981; Conrad et al., 1992). NAPL is trapped by a mechanism known as "bypassing" in regions with several adjacent large pore bodies connected by relatively large throats and surrounded by smaller throats, such as would be found in a lens of coarse sand within a finer sand (Morrow, 1971). The actual geometry of blobs generated by this mechanism in a real porous medium is very complex, often encompassing several adjacent pore bodies and throats (Chatzis et al., 1983; Schwille, 1988; Conrad et al., 1992). Chatzis et al. (1983) provide a thorough description of NAPL entrapment mechanisms.

The actual distribution of NAPL blob sizes has been studied with the use of styrene which is solidified within the pore spaces (Morrow and Chatzis, 1981; Chatzis et al., 1983; Conrad et al., 1992; Mayer and Miller, 1992). This aromatic hydrocarbon is a reasonable choice for solidification because its fluid properties (interfacial tension, density, viscosity) are similar to properties of NAPL's encountered at contaminated groundwater sites. After styrene is entrapped within a porous medium, it is subjected to conditions promoting polymerization reactions, resulting in the formation of solid polystyrene blob casts. Analysis of the solidified blobs is generally accomplished by categorizing them into sets of similarly sized blobs for analysis by computer-aided image techniques to determine blob size distributions. Chatzis et al. (1983) showed that a majority of blobs $(\sim 65 \%)$ produced in an unconsolidated, uniform-size distribution sand pack occupied only one pore body, while $\sim 15 \%$ of the blob population were branched. The length and volume distribution of styrene blobs entrapped in three different uniform size fractions of glass beads were measured by Mayer and 
Miller (1992). They found that the median blob length was correlated to the median glass bead size and that the distribution of blob sizes was much wider than the distribution of pore sizes in each of the media.

\subsection{NAPL dissolution}

Entrapped NAPL blobs may dissolve into groundwater, creating a long-term source of contamination. Because this entrapped organic phase is difficult to remove from the pore spaces by hydraulic forces (J.L. Wilson and Conrad, 1988), it is necessary to understand the phenomena controlling the dissolution process. Critical to the long-term dissolution process is the decrease in NAPL-water surface area over time as the volume of the entrapped NAPL is reduced through dissolution. Powers et al. (1994) showed that, as the NAPL blobs shrank, effluent solute concentrations from column dissolution experiments decreased to levels orders of magnitude less than equilibrium concentrations. These concentrations, however, exceeded drinkingwater quality limits for a period of time much greater than would be predicted by equilibrium considerations.

A number of mathematical models describing rate-limited NAPL dissolution from entrapped NAPL blobs have been proposed in the literature. These generally have the form of a linear-driving-force expression (e.g., Hunt et al., 1988; Miller et al., 1990; Powers et al., 1991). The flux of a species (i) between phases is approximated as the product of a mass-transfer coefficient $\left(k_{f i}\right)$ and the difference between its bulk aqueous-phase concentration $\left(C_{i}\right)$ and that concentration which would be in equilibrium with its concentration in the organic phase $\left(C_{\mathrm{si}}\right)$ (Weber, 1972). The total mass transferred is the product of this flux and the specific interfacial area across which mass transfer can occur:

$$
S_{i}=k_{\mathrm{ri}} a_{\mathrm{o}}\left(C_{\mathrm{s} i}-C_{i}\right)
$$

where $S_{i}$ is the solute mass transferred between phases per unit time and volume of the medium; and $a_{0}$, the specific surface area, is the NAPL-water interfacial area per unit volume of medium. Over time, the interphase flux may decrease as the mass-transfer coefficient, specific surface area, or equilibrium concentration decrease. These changes must be quantified in order to accurately model the transient NAPL dissolution process. In single-component organic-phase systems, the specific surface area is the primary transient variable in the source term. The specific surface area can decrease by orders of magnitude as the organic solute is removed from the system and the volume of entrapped NAPL is reduced.

Researchers who employed a linear-driving-force relationship as a basis for transient NAPL dissolution models have typically used expressions for lumped masstransfer coefficients $(\hat{k})=k_{\mathrm{f}} a_{\mathrm{o}}$ ) so that the mass-transfer coefficient and specific surface area do not need to be determined independently (Miller et al., 1990; Imhoff et al., 1994; Powers et al., 1994). In these models, the volumetric fraction of NAPL was used in correlations for lumped mass-transfer coefficients as a surrogate indicator of the specific interfacial area across which mass transfer can occur.

Powers et al. (1994) showed that the relationship between decreasing volumetric 
fraction and reduced mass-transfer rate depends on characteristics of the porous medium. This relationship was adequate for the prediction of the overall decrease in effluent solute concentrations from NAPL dissolution in one-dimensional sand columns. However, the predicted effluent concentration curves did not mimic the shape of the measured concentration curve. The variations in the shape of the concentration-time profiles were attributed to the removal of various size fractions of entrapped blobs.

Imhoff et al. (1994) noted that the correlation between NAPL volumetric fraction and mass-transfer rate was not sufficient to describe the decreasing mass-transfer rates observed over time. Later analyses (Imhoff et al., 1993) suggested that variations in the relative permeability, and therefore the interstitial velocity, across the column resulted in increased channeling within the column as time progressed. The formation of "dissolution fingers" under these conditions created heterogeneous mass-transfer rates along the column length.

The linear-driving-force mass-transfer model utilizing a lumped mass-transfer coefficient has been extended to incorporate the effects of solute diffusion through zones of immobile water (Robinson and Bedient, 1991; Borden and Kao, 1992). This model, referred to as the "two-site model", assumes that a fraction of NAPL is in equilibrium with the aqueous phase; and the remaining portion, being trapped in inaccessible pores, is subject to rate-limited mass transfer as the organic species diffuse through the immobile water. Two parameters are required to define the overall dissolution rate for this type of model, a lumped mass-transfer coefficient and the fraction of NAPL in equilibrium with the aqueous phase. In their studies, data from the leaching of gasoline entrapped in a sandy medium were used to calibrate the twosite model. Due to the very low mass fractions of many of the components in gasoline, it was assumed that the composition, but not specific surface area, changed over the course of the experiments. The parameters required for this model were determined by fitting the model to experimental data; no method of estimating these parameters from physical properties of the porous medium or NAPL properties are presently available.

A variation of the two-site model has also been used to describe the retardation of solutes as they partition from the aqueous phase into immiscible NAPL blobs (Brusseau, 1992; Hatfield et al., 1993). Use of the two-site model in these applications also involved the assumption that phase compositions, but not interfacial areas, change with time. Hatfield et al. (1993) considered rate-limited exchange of solutes between the aqueous and solid phase as well as the organic and aqueous phases, where the aqueous phase included both mobile and immobile water zones. By comparing this complex model to column dissolution data, it was concluded that the NAPLmobile water interphase mass-transfer rates were the rate-limiting process in the medium-fine uniform sand column studied. Brusseau (1992) stated that the twosite model fits experimental data when rate parameters were determined from independent data sets. Two-site models have not been compared with data sets which involve time-dependent NAPL-water interfacial areas.

A third approach to the mathematical modeling of the transient process of NAPL dissolution from entrapped blobs involves the independent quantification of the 
mass-transfer coefficient and specific surface area appearing in Eq. 1. deZabala and Radke (1986), Hunt et al. (1988) and Powers et al. (1991) utilized this approach in theoretical evaluations of the significance of rate-limited dissolution processes. These researchers assumed an idealized geometry of NAPL blobs in order to estimate the specific surface area. Conclusions drawn from these assessments regarding the applicability of the local equilibrium assumption have been shown to be very sensitive to the assumed NAPL distribution (Hunt et al., 1988; Powers et al., 1991). deZabala and Radke (1986) assumed that blobs were distributed as very tiny spheres $(0.001 \mathrm{~cm})$, and concluded that mass-transfer processes were not rate limited. Hunt et al. (1988) considered NAPL globules distributed as spheres with diameters ranging from 0.1 to $1000 \mathrm{~cm}$, and concluded that rate-limited mass transport would be very significant for the larger globule sizes. Mass-transfer coefficients for these studies were generally estimated from correlations developed for the dissolution of packed beds of naphthalene spheres (e.g., E.J. Wilson and Geankoplis, 1966), even though the hydrodynamics in such a system would be expected to differ from the flow of water around sand grains and amorphous NAPL blobs.

In an analysis of a set of one-dimensional NAPL dissolution experiments, Geller and Hunt (1993) assumed that NAPL blobs could be modeled as a set of mono-sized spheres. Mass-transfer coefficients calculated from the E.J. Wilson and Geankoplis (1966) correlation . ere assumed to be appropriate. The initial diameter of the NAPL spheres was used to calibrate the model. Initial diameters were found to range from 3.5 to 10 times the glass-bead diameter used as a porous medium. It was noted that this range was within the range of blob sizes determined by the analysis of polystyrene blob distributions (Chatzis et al., 1983). The calibrated model provided a very good representation of the column effluent concentrations over the approximate range of $0.35<C / C_{\mathrm{s}}<0.01$. Concentration data were nol pivited on a log scale so it was difficult to assess the adequacy of this model at low concentrations.

Although the sphere model presented by Geller and Hunt (1993) was adequate for the data used in its development, it did not account for the range of blob sizes which are entrapped within porous media of environmental significance. Conrad et al. (1992) hypothesized that this distribution of blob sizes would affect NAPL dissolution rates. Powers et al. $(1992,1994)$ showed that this is indeed the case; both steady-state and transient NAPL dissolution rates measured in one-dimensional columns were dependent on NAPL blob distributions. The net mass-transfer rates were observed to be slower for the large or branched ganglia entrapped in coarse or graded sands, respectively, due to the decreased surface area.

In this paper, an experimentally-based phenomenological model for transient NAPL dissolution is developed. This model attempts to account for the complex distribution of NAPL blobs entrapped within the saturated zone. It extends the geometrical modeling approach discussed above by assuming that the entrapped NAPL can be better represented by a range of spherical blob sizes rather than mono-sized spheres. To develop a phenomenological model, experimental data are required for independent characterization of the NAPL-water specific surface area and mass-transfer coeficients appearing in Eq. 1. In the proposed model, the specific 
Table 1

Experimental variables for naphthalene dissolution experiments

\begin{tabular}{lll}
\hline Experimental variable & Symbol & Range of values \\
\hline Superficial aqueous-phase velocity $\left(\mathrm{m} \mathrm{day}^{-1}\right)$ & $q$ & $0.17-17$ \\
Naphthalene volumetric fraction $(-)$ & $\theta_{n}$ & $0.015-0.088$ \\
Naphthalene sphere diameter $(\mathrm{cm})$ & $d_{s}$ & $0.22-0.49$ \\
Median sand grain diameter $(\mathrm{cm})$ & $d_{50}$ & $0.045-0.17$ \\
\hline
\end{tabular}

surface area is approximated by treating entrapped NAPL blobs as a set of spheres with a range of diameters. Diameter distributions are determined through the entrapment, polymerization and characterization of styrene blobs in sandy media. Masstransfer coefficients for this model are estimated from experimental measurements of the dissolution of naphthalene when emplaced as solid spheres within a sandy medium. One-dimensional column experiments, described by Powers et al. (1994), were conducted to measure the effluent concentrations of styrene or trichloroethylene (TCE) over time as the entrapped NAPL dissolved. These data are used to calibrate and verify the phenomenological model.

\section{Experimental methods}

\subsection{Naphthalene sphere dissolution}

To estimate mass-transfer coefficients, a set of controlled specific surface area dissolution experiments were undertaken. The use of solid naphthalene spheres as the dissolving organic phase emplaced within a sandy medium allowed the quantification of mass-transfer coefficients in a system with known interfacial area. Porous medium characteristics, aqueous-phase velocity, bed length, naphthalene volumetric fraction and sphere diameter were varied in this set of experiments (Table 1). The hydrodynamics of this naphthalene-sand system were assumed to be more similar to those of water flow through sandy media containing entrapped NAPL than the hydrodynamics of a uniformly packed bed of large dissolving spheres. The latter system was the basis for available mass-transfer correlations such as that developed by E.J. Wilson and Geankoplis (1966).

The experimental design required the production of naphthalene spheres with a range of diameters. Reagent-grade crystalline naphthalene (Fischer Scientific $\left.{ }^{(\hat{\hat{B}}}\right)$ was melted $\left(110^{\circ} \mathrm{C}\right)$ and formed into spheres by dropping the molten chemical from a capillary pipette through a $40-\mathrm{cm}$ tall water column with a temperature decrease $\left(85^{\circ}\right.$ to $10^{\circ} \mathrm{C}$ ) over its length. The hot water at the top of the column allowed the molten naphthalene to assume a spherical shape as it slowly dropped through the water. The spheres solidified as they settled through the colder water at the bottom of the column. The solid spheres were gently sieved to segregate them into sets of equal size. Three different sets of spheres were produced with diameters ranging from 0.22 to $0.49 \mathrm{~cm}$. Spheres smaller than this were not used because they sublimated rapidly, 
Table 2

Sand grain-sıze che racterist: is

\begin{tabular}{lll}
\hline Sal'ú iype & $\begin{array}{l}d_{\text {so }_{\mathrm{s}}} \\
(\mathrm{cm})\end{array}$ & $U_{1}^{\mathrm{a}}$ \\
\hline Wagner \#50 & 0.045 & 1.45 \\
Ottuwa & 0.071 & 1.21 \\
Wagner \#18 & 0.120 & 1.19 \\
Wagner \#14 & 0.170 & 1.21 \\
Wagner Mix \#l & 0.080 & 2.42 \\
\hline
\end{tabular}

'Uniformity index is a measure of grain-size distribution $\left(U_{i}=d_{60} / d_{10}\right)$.

resulting in a variable surface area. A representative sample of each size fraction was weighed and counted. The naphthalene surface area was then calculated assuming that perfectly spherical shapes were produced.

Two general types of soils were employed in all experiments: Ottawa sand, a uniform (0.058-0.084 cm; \#20-\#30 mesh) silica sand purchased from Fisher Scientific ${ }^{\circledR}$; and Wagner soil, collected frem a gravel pit in Ann Arbor, Michigan, U.S.A. The Wagner soil is typical of aquifer materials in the Michigan area, which are predominantly of glacial origin. The Wagner soil was sieved into uniform size fractions and washed with deionized water prior to use. All size fractions were comprised of very angular grains, representing a wide range of mineral components. Fractions retained on the \#14 $(0.14-0.20 \mathrm{~cm})$, \#18 $(0.1-0.14 \mathrm{~cm})$ and \#50 $(0.03-0.06 \mathrm{~cm})$ sieves were used as uniform sand isolates. A mixture of the sieved sand fractions was also used (Wagner Mix \#I). The grain-size distributions of these media are characterized in Table 2 by their median grain size $\left(d_{50}\right)$ and uniformity index $\left(U_{i}=d_{60} / d_{10}\right)$ (Driscoll, 1986). Here, $d_{x x}$ is the diameter of the particles which have $x x \%$ of the particles, by weight, smaller than this size.

The stainless-steel and glass columns described in Powers et al. (1992) were used for naphthalene sphere dissolution experiments. During column packing, a known mass of spheres was distributed throughout the column, as dry sand was added under vibration. The emplaced volumetric fraction of naphthalene was calculated from the density of naphthalene and the column volume. De-aired water was pumped through the column to dissolve any entrapped air. Because naphthalene has a very low solubility ( $30 \mathrm{mg} \mathrm{L}^{-1}$; Verschueren, 1983), an insignificant amount of naphthalene was removed from the column during this procedure. Water was then pumped through the column at a constant rate and effluent samples were collected under steady-state conditions. An average of seven aqueous-phase velocities were used for each experiment. Eighteen column experiments were conducted, generating a total of 128 data points for use in the mathematical model development.

Concentration measurements from aqueous-phase effluent samples were used to compute mass-transfer coefficients. Effluent samples were collected in $2-\mathrm{mL}$ headspace-free serum vials and immediately capped with Teflon ${ }^{(\mathfrak{k})}$-lined aluminum crimp caps. Triplicate samples were collected and analyzed for each velocity during the steady-state period. Samples were analyzed by high-pressure liquid chromatography 
(HPLC) to determine naphthalene solute concentrations. The HPLC system consisted of a Waters ${ }^{\left({ }^{(}\right)}$M45 solvent pump, Waters ${ }^{(\mathfrak{k})}$ Lambda Max. variable-wave-length LV spectrophotometer (Model 480) and Hewlett-Packard 'iq) 3390A plotter integrator. A $70 \%$ methanol- $30 \%$ water mobile phase and a detector wave length of $254 \mathrm{~nm}$ were employed.

\subsection{NAPL dissolution experiments.}

NAPL dissolution experiments were conducted to generate data for model calibration and verification. The procedures used and results obtained are described in detail by Powers et al. (1994) and are briefly summarized here.

Two organic phases, styrene (Aldrich Chemical $\left.{ }^{(i)}\right)$ and TCE (Fischer Scientific ${ }^{(\hat{i})}$ ) were employed in the column dissolution experiments. A hydrophobic dye, Oil-Red$\mathrm{O}^{(\mathfrak{i n})}$ (Fischer Scientific $\left.{ }^{(\hat{i})}\right)$, was added to both NAPL's $\left(0.5 \mathrm{~g} \mathrm{~L}^{-1}\right)$ so that flow and distribution patterns within the column could be qualitatively observed. Porous media employed for this set of experiments were identical to those described for the naphthalene dissolution experiments (Table 2).

NAPL dissolution rates were measured in custom-designed and -manufactured one-dimensional soil columns $(5.5 \mathrm{~cm}$ diameter $\times 3-4 \mathrm{~cm}$ long $)$ as described by Powers et al. (1992). After saturating the sand with de-aired water, a series of immiscible displacements was used to obtain a relatively uniform distribution of entrapped NAPL biobs throughout the column. Water was then pumped through the column at a constant velocity and effluent samples were collected over an extended period of time (2-6 days). These sampies were diluted in methanol and analyzed with the HPLC equipment described above to determine aqueous-phase concentrations of the organic species.

\subsection{Characterization of NAPL distribution}

Styrene polymerization techniques were used to characterize the NAPL blob size distributions. The procedure described above for the entrapment of styrene by immiscible displacement of the fluid phases was used to obtain a residual saturation of entrapped NAPL throughout the column. The columns were then subjected to high temperatures $\left(80^{\circ} \mathrm{C}\right)$ and pressures $(5.5 \mathrm{bar})$ for $\sim 48 \mathrm{~h}$, during which time the styrene was completely polymerized. Visual observation of the sand-polystyrene mixture as it was removed from the column indicated that the polystyrene blobs were distributed throughout the column in a relatively uniform manner. Siparation of the polystyrene blobs from the sand was subsequently accomplished in several steps. Large multipore space blobs oí polystyrene encompassing many sand grains were separated by sieving through a coarse $(0.1-0.2 \mathrm{~cm})$ screen. Singlet globules were separated gravimetrically in a $50 \%$ solution of potassium bicarbonate having a density $\left(1.5 \mathrm{~g} \mathrm{~cm}^{-3}\right)$ intermediate between sand grains and singlets. Sand grains enmeshed within NAPL blobs were dissolved in sequential baths of sulfuric acid, hydrofluoric acid, hydrochloric acid and a 30\% hydrogen peroxide solution. Separated polystyrene blobs were sorted into fractions of similar size by 
careful hand-sieving. Each size fraction was weighed and a representative mass of blobs counted to determine the number and mass distribution of blobs. Because styrene shrinks by as much as $17 \%$ upon polymerization (Boundy et al., 1965), the solid polystyrene blobs were slightly smaller than their liquid counterparts.

\section{Experimental results and analyses}

\subsection{Naphthalene sphere dissolution}

Results of naphthalene dissolution experiments consisted of effluent naphthalene concentrations for a range of experimental conditions. These data were used to develop a correlation expression for the mass-transfer coefficients as a function of system parameters. First, mass-transfer coefficients, $k_{f}$, were calculated from column effluent data by employing a solution of the steady-state solute transport equation. For this type of system, it has been shown that dispersion has negligible impact on calculated mass-transfer coefficients (Wakao and Funazkri, 1978). Assuming that dispersion can be neglected, the steady-state transport equation may be written as:

$$
q \frac{\mathrm{d} C}{\mathrm{~d} x}=k_{\mathrm{f}} a_{\mathrm{o}}\left(C_{\mathrm{s}}-C\right)
$$

where $q$ is the superficial aqueous-phase velocity; $C_{\mathrm{s}}$ is the solubility of naphthalene in water; and $x$ is the length along the column. Subject to a first-type boundary condition at the column inlet $(C=0$ at $x=0)$, this equation can be solved and rearranged to yield an expression for the mass-transfer coefficient, $k_{\mathrm{r}}$ :

$$
k_{\mathrm{f}}=-\frac{q}{a_{\mathrm{o}} L} \ln \left(1-C / C_{\mathrm{s}}\right)
$$

Here $L$ is the column length; $C$ is the effluent concentration; and $a_{0}$, the specific surface area, is computed from the spherical geometry of the emplaced organic phase, $a_{\mathrm{o}}=\theta_{\mathrm{n}}\left(6 / d_{\mathrm{s}}\right)$, where $d_{\mathrm{s}}$ is the diameter and $\theta_{\mathrm{n}}$ is the volumetric fraction of the naphthalene spheres.

Mass-transfer coefficients computed from Eq. 3 were then incorporated into a dimensionless Sherwood number. This number, defined as:

$$
\mathrm{Sh}=k_{\mathrm{f}} I_{\mathrm{c}} / D_{\mathrm{L}}
$$

where $I_{\mathrm{c}}$ is a characteristic length; and $D_{\mathrm{L}}$ is the free liquid diffusivity of the solute, relates the resistances due to interphase mass transport to those of molecular diffusion (Welty et al., 1969). A correlation was sought which would relate the Sherwood number to dimensionless groups describing independent system parameters. An extensive number of Sherwood number correlations for dissolution in packed beds have appeared in the chemical engineering literature (e.g., Treybal, 1980). In these correlations, dimensional analysis has lead to the incorporation of independent variables, including characteristic length, aqueous-phase velocity, fluid density $(\rho)$ 
and viscosity $(\mu)$ and solute-free liquid diffusivity, into dimensionless Reynolds and Schmidt numbers. Here, the Reynolds number,

$$
\operatorname{Re}=q \rho_{w} l_{c} / \mu_{w}
$$

is the ratio of inertial forces to viscous forces, and the Schmidt number,

$$
\mathrm{Sc}=\mu_{\mathrm{w}} /\left(D_{\mathrm{L}} \rho_{\mathrm{w}}\right)
$$

is a ratio of viscous to molecular diffusional forces. In order to account for changes in pore-water velocity as NAPL mass is removed from the system, Reynolds numbers based on both superficial aqueous-phase velocities $(\mathrm{Re})$ and interstitial velocities:

$$
\operatorname{Re}^{\prime}=q \rho_{w} l_{c} /\left[\mu_{w}\left(\epsilon-\theta_{n}\right)\right]
$$

where $\epsilon$ is the porosity, were considered in the development of Sherwood number correlations in the present study. Two alternative characteristic lengths were used for the Reynolds and Sherwood numbers: the median sand grain diameter and the naphthalene sphere diameter. The Schmidt number was not varied in this set of experiments and, thus, could not be directly incorporated into the Sherwood number correlation.

The complex geometry of the entrapped NAPL blobs could also affect aqueousphase hydrodynamics in the sandy media and lead to a dependence of the organicphase dissolution rate on blob geometry. Thus, additional dimensionless groups were also considered in the development of the Sherwood number correlation. These included the volumetric fraction of the organic phase and a ratio of the sphere diameter to the median grain diameter $\left(d_{\mathrm{s}} / d_{50_{\mathrm{g}}}\right)$. The absolute size of the sand grain and naphthalene sphere diameters were also considered by normalizing these to a "medium" sand grain diameter $\left(d_{M}=0.05 \mathrm{~cm}\right)$, as defined by the U.S. Department of Agriculture (Driscoll, 1986). The normalized median grain diameter is given as $\delta_{\mathrm{g}}=d_{50_{\mathrm{g}}} / d_{\mathrm{M}}$ and the normalized naphthalene sphere diameter as $\delta_{\mathrm{s}}=d_{\mathrm{s}} / d_{\mathrm{M}}$. It was assumed that correlations of the form:

$$
\mathrm{Sh}=\gamma \operatorname{Re}^{\beta_{1}} \prod_{i=2}^{n}\left(P_{i}\right)^{\beta_{1}}
$$

or

$$
\mathrm{Sh}=\gamma \operatorname{Re}^{\beta^{\beta_{1}}} \prod_{i=2}^{n}\left(P_{i}\right)^{\beta_{1}}
$$

would be appropriate for this mass-transfer process. Here, the $P_{i}$ 's are an unknown subset of the independent dimensionless variables mentioned above; and $\gamma$ and $\beta_{i}$ are parameters to be determined. Due to the exclusion of the Schmidt number, correlations of this form are limited to dissolution of compounds with Sshmidt numbers similar to that of naphthalene $(\mathrm{Sc}=1250)$.

A stepwise regression technique (Walpole and Myers, 1989) was used to systematically determine the best combination of independent parameters for inclusion in the correlation to express the Sherwood numbers for the naphthalene dissolution experiments. This procedure involves the stepwise addition of parameters to the 
Table 3

Correlations developed for naphthalene dissolution experiments

\begin{tabular}{lllll}
\hline $\begin{array}{l}\text { Equation } \\
\text { No. }\end{array}$ & $\mathrm{Sh}=\gamma \mathrm{Re}^{\beta_{1}}$ & $\begin{array}{l}\text { Coefficient of } \\
\text { determination, } R^{2}\end{array}$ & $\begin{array}{l}95 \% \text { Confidence } \\
\text { interval for } \gamma\end{array}$ & $\begin{array}{l}95 \% \text { Confidence } \\
\text { interval for } \beta_{1}\end{array}$ \\
\hline Sa & $S h=77.6 \mathrm{Re}^{0.658}$ & 0.86 & \pm 1.2 & \pm 0.052 \\
$5 \mathrm{~b}$ & $\mathrm{Sh}=36.8 \mathrm{Re}^{0.654}$ & 0.85 & \pm 1.1 & \pm 0.052 \\
\hline
\end{tabular}

model in an order based on the values of the regression coefficients of the individual parameters. After addition of each parameter, the value of the additional parameter is assessed and the continued value of previously added parameters is ensured. The coefficient of multiple determination $\left(R^{2}\right)$ is used to assess the added value of the additional component, while a $t$-test is used to ensure that each of the parameters in the model have statistical significance. The best-fit model was defined as that combination of parameters which maximized the $R^{2}$ statistic, while minimizing the standard error of the estimate and ensuring that the $t$-statistic for each coefficient was greater than critical values of the two-sided $t$-distribution for a statistical significance level of $\alpha=0.01$. For each definition of the Reynolds number, this process was repeated twice, once utilizing the medien sand grain diameter as the characteristic length, and once with the sphere diameter as the characteristic length. Results of the regression analysis indicated that models based on median sand grain diameter as the characteristic length were superior to those based on sphere diameters. "Superior" here implies higher $R^{2}$ statistics and lower values of the standard error of the estimate. It was also determined that the Sherwuod numbers were not correlated to the volumetric fraction nor to the ratio of sphere to grain sizes. There was a weak correlation between the Sherwood number and either the normalized median grain diameter or sphere size. However, an increase in the $R^{2}$ statistic with the addition of any parameter would be anticipated and thus, these weak correlations were not considered statistically significant.

The correlation expressions for the naphthalene dissolution experiments which were generated from the stepwise multiple regression analysis are presented in Table 3:

$$
\begin{aligned}
& \mathrm{Sh}=77.6 \operatorname{Re}^{0.658} \\
& \mathrm{Sh}=36.8 \mathrm{Re}^{0.654}
\end{aligned}
$$

Note that there is virtually no statistical difference between the correlation based upon superficial velocity (Eq. 5a) and the correlation based upon interstitial velocity (Eq. 5b). Fig. 1 is a plot of the experimental Sherwood numbers for naphthalene sphere dissolution vs. Reynolds number. The solid line represents the correlation given by Eq. $5 \mathrm{a}$.

The developed correlations (Eqs. 5a and 5b) can be compared with others presented in the literature for a wide range of engineered systems (Table 4). These published correlations differ due to variability in the characteristics of the system of interest and in the methods used to calculate mass-transfer coefficients from experimental data. 


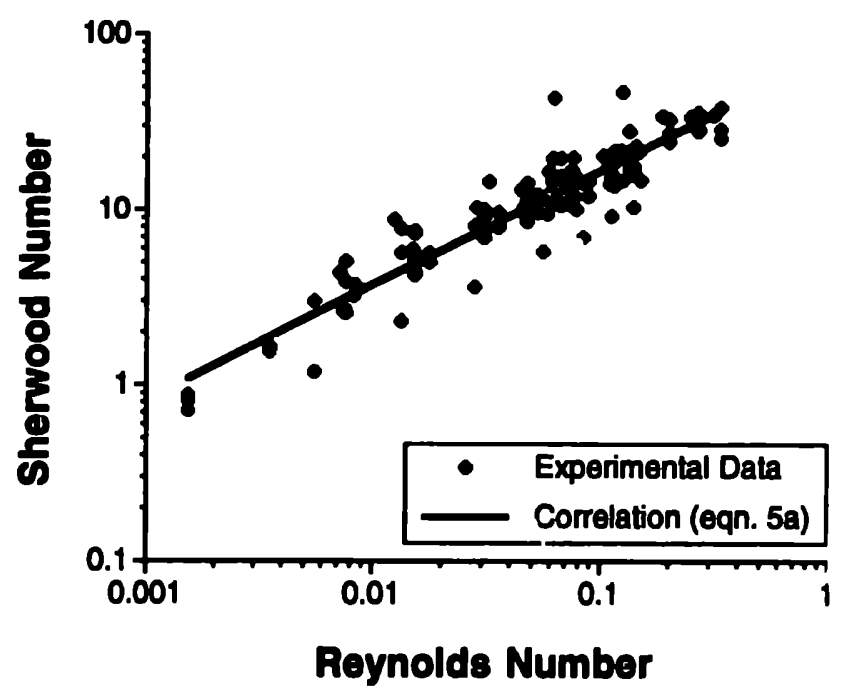

Fig. 1. Results from naphthalene sphere dissolution experiments compared with Sherwood numbers estimated from Eq. 5 a.

Fig. 2 illustrates that Sherwood numbers predicted by several correlations which could be considered analogous to the dissolution of NAPL's entrapped within porous media vary over orders of magnitude. Some of this variation has been attributed to:

Table 4

Relationships between Sherwood number and Reynolds number

\begin{tabular}{|c|c|c|c|}
\hline Conditions & $\beta_{1}^{a}$ & Valid for & Reference \\
\hline \multicolumn{4}{|l|}{ Single spheres: } \\
\hline $\begin{array}{l}\text { Fluid sphere } \\
\text { Solid sphere }\end{array}$ & $\begin{array}{l}0.50 \\
0.33\end{array}$ & $\begin{array}{l}\operatorname{Re}>5 \\
\operatorname{Re} \cdot \mathrm{Sc}>1000\end{array}$ & $\begin{array}{l}\text { Ranz (1952) } \\
\text { Friedlander (1957) }\end{array}$ \\
\hline \multicolumn{4}{|l|}{ Packed beds: } \\
\hline Solid spheres, low $\mathbf{R e}$ & $\begin{array}{l}0.33 \\
0.28\end{array}$ & $\begin{array}{l}0.0016<\operatorname{Re}<55 \\
\operatorname{Re}<10\end{array}$ & $\begin{array}{l}\text { E.J. Wilson and Geankoplis (1966) } \\
\text { Dwivedi and Upadhyay (1977) }\end{array}$ \\
\hline Spheres emplaced in sand & 0.66 & $0.001<\operatorname{Re}<0.33$ & this wort \\
\hline Dense packings, fine media & 1.00 & $0.01<\operatorname{Re}<100$ & Nelson and Galloway (1975) \\
\hline Solid spheres, higher $\mathbf{R e}$ & $\begin{array}{l}0.69 \\
0.56 \\
0.60\end{array}$ & $\begin{array}{l}\operatorname{Re}>55 \\
\operatorname{Re}>10 \\
3<\operatorname{Re}<10,000\end{array}$ & $\begin{array}{l}\text { E.J. Wilson and Geankoplis (1966) } \\
\text { Kumar et al. (1977) } \\
\text { Wakao and Funazkri (1978) }\end{array}$ \\
\hline NAPL dissolution: & & & \\
\hline $\begin{array}{l}\text { Oil pancake } \\
\text { Residual blobs }\end{array}$ & $\begin{array}{l}1.50 \\
0.61 \\
0.75 \\
0.87\end{array}$ & $\begin{array}{l}0.65<\operatorname{Re} . \mathrm{Sc}<100 \\
0.01<\operatorname{Re}<0.3 \\
0.0015<\operatorname{Re}<0.03 \\
0.0006<\operatorname{Re}<0.006\end{array}$ & $\begin{array}{l}\text { Pfannkuch }(1984) \\
\text { Powers et al. }(1991)^{b} \\
\text { Miller et al. }(1990)^{b} \\
\text { Imhoff et al. }(1994, \text { model } l)^{\text {b }}\end{array}$ \\
\hline
\end{tabular}

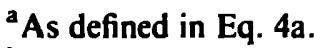

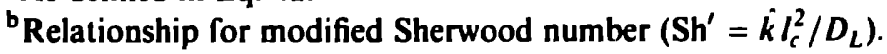




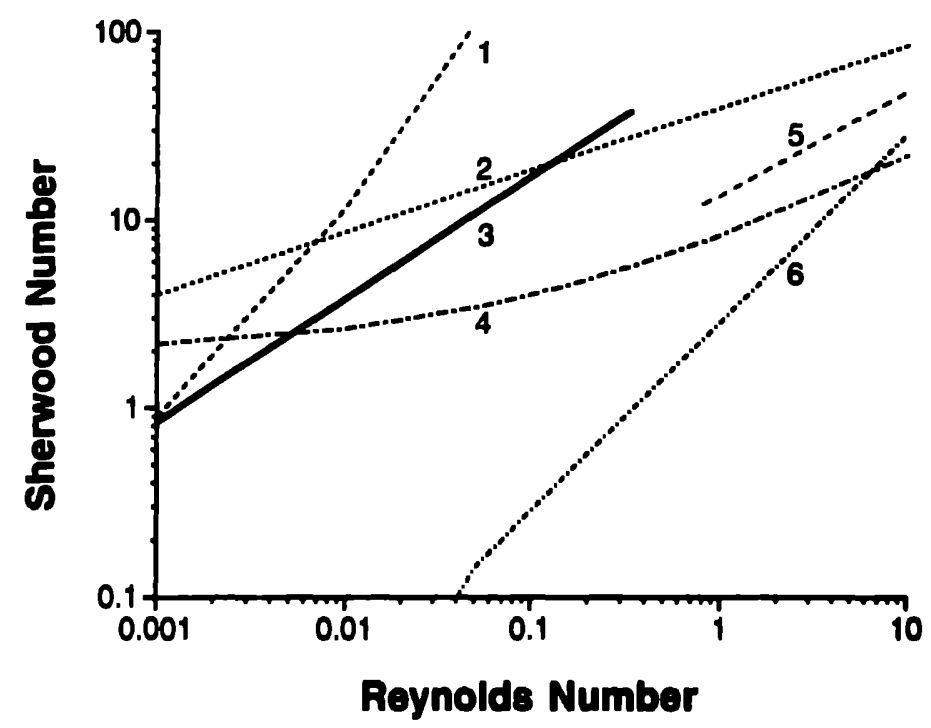

Fig. 2. A comparison of Sherwood numbers calculated from several correlations for interphase masstransfer processes $[I=$ Pfannkuch (1984); $2=$ E.J. Wilson and Geankoplis (1966); $3=$ this work (Eq. 5a); $4=\operatorname{Ranz}(1952) ; 5=$ Wakao and Funazkri (1978); $6=$ Nelson and Galloway (1975)].

(1) reduced mass-transfer resistance in liquid-liquid systems in comparison with mass transfer from solid spheres due to internal circulation (Bowman et al., 1961);

(2) the generation of two-dimensional systems due to axial diffusion in gas-phase, packed-bed systems (Wakao and Funazkri, 1978);

(3) differences in the definition of the mass-transfer coefficient; both "effective" mass-transfer coefficients and local mass-transfer coefficients have been used (Fedkiw and Newman, 1978); and

(4) the particle size of the porous medium; lower mass-transfer rates have been measured in fine media (Nelson and Galloway, 1975).

Another way to compare these correlations is to consider the exponent of the Reynolds number $\left(\beta_{1}\right)$. This exponent quantifies the relationship between the Sherwood number and the Reynolds number. Table 4 summarizes the values of the $\beta_{1}$ coefficient used in systems similar to the dissolution of NAPL blobs. Coefficients in this table include both theoretically derived and experimentally determined values. It can be noted that exponent values fit to the naphthalene sphere dissolution data $\left(\beta_{1}=0.66\right)$ are similar to those published for entrapped NAPL dissolution studies $\left(\beta_{1}=0.61-1.5\right)$ and are consistently higher than those developed for the dissolution of packed beds of uniform-sized spheres at low velocities $\left(\beta_{1}=0.28-0.33\right)$. The exponents determined in the present study are, however, consistent with the published $\beta_{1}$-values for packed-bed systems at higher Reynolds numbers $\left(\beta_{1}=0.56\right.$ 0.69).

The large variability in Sherwood number magnitude and dependence on the Reynolds number among these correlations emphasizes the need for careful selection of a mass-transfer coefficient correlation based upon consideration of the similarity between the conditions and equations used for their development and those of the system under study. Correlations developed for the dissolution of packed beds containing relatively large $(>0.5 \mathrm{~cm})$ dissolving spheres at low Reynolds numbers (e.g., 
E.J. Wilson and Geankoplis, 1966) and correlations developed for the dissolution of single spheres suspended in a flowing fluid (Bowman et al., 1961) have been used to analyze NAPL blob dissolution processes in previous studies (Hunt et al., 1988; Powers et al., 1991; Geller and Hunt, 1993). The above discussion indicates, however, that these systems are probably not analogous to NAPL dissolution scenarios and the use of such correlations may be inappropriate under some conditions. In the present study, the $\beta_{1}$ exponent in the correlation for naphthalene sphere dissolution is similar to exponents for NAPL dissolution presented in several other studies (Table 4). Thus, it is probable that the hydrodynamics of the system considered herein are similar to those encountered in systems involving NAPL blob dissolution.

\subsection{Distribution of entrapped NAPL}

The distribution of NAPL blob sizes entrapped within a porous medium was determined by the entrapment, polymerization and characterization of styrene blobs in four different sandy media. The Ottawa, Wagner \#18 and Wagner \#50 sands were comprised of uniform sand grain-size distributions, while the Wagner Mix \#I was comprised of a wider distribution of sizes (Table 2). NAPL blobs entrapped in these sands included a wide range of shapes and sizes. Smaller blobs occupying one pore space, "singlets", were spherical, while the larger ganglia were amorphous. Several of these were long cylindrical shapes while others had multiple "fingers" extended in many directions. Powers et al. (1992) present photographs illustrating the range of NAPL blob shapes and sizes entrapped in sandy media.

Sieving the polystyrene blobs into separate size fractions enabled evaluation of the size distributions. Examples of the polystyrene blob number and mass frequency distributions for the uniform Wagner \#50 and graded Wagner Mix \#l sands are presented in Fig. 3a and b, respectively. Distribution plots for other sands can be found in Powers (1992). Frequency distribution plots show that a greater number of blobs are entrapped as small blobs (singlets), with more mass typically distributed in the form of larger ganglia. The range of size distributions, however, depends upon the particular porous medium. The greatest difference between number and mass distributions occurred in the graded Wagner Mix \#l sand (Fig. 3b). A few large blobs entrapped in this sand account for nearly $30 \%$ of the total NAPL volume. Inspection of polymerized blobs suggested that the small singlets have much greater exposed surface area per unit volume than the very large and amorphous ganglia.

In an effort to quantify the size distribution of NAPL blobs, an "effec:Ive" diameter was calculated for each sieved fraction of blobs. For this calculation, an effective sphere was defined as that sphere which has the same volume $\left(V_{\mathrm{b}}^{j}\right)$ as the average blob in that sieve fraction:

$$
V_{\mathrm{b}}^{j}=\frac{m_{\mathrm{b}}^{j}}{\rho_{\text {sty }} N_{\mathrm{b}}^{j}}=\frac{4}{3} \pi\left(\frac{d_{\mathrm{b}}^{j}}{2}\right)^{3}
$$

where $d_{\mathrm{b}}^{j}$ is the effective sphere diameter for blobs retained on sieve $j: m_{\mathrm{b}}^{j}$ is the mass of 
Table 5

Summary of effective blob diameters

\begin{tabular}{|c|c|c|c|c|c|c|}
\hline \multirow[t]{2}{*}{ Sand type } & \multicolumn{3}{|c|}{ Mass distribution } & \multicolumn{3}{|c|}{ Number distribution } \\
\hline & $\begin{array}{l}d_{s_{\mathrm{b}}} \\
(\mathrm{cm})\end{array}$ & $d_{50_{b}} / d_{50_{\mathrm{b}}}$ & $U_{i}$ & $\begin{array}{l}d_{s a_{b}} \\
(\mathrm{~cm})\end{array}$ & $d_{S_{b}} / d_{S_{0,}}$ & $U_{i_{b}}$ \\
\hline Wagner \#50 & 0.068 & 1.51 & 1.9 & 0.041 & 0.91 & 1.4 \\
\hline Otlawa & 0.088 & 1.24 & 2.2 & 0.058 & 0.82 & 3.1 \\
\hline Wagner \#18 & 0.120 & 1.00 & 2.0 & 0.073 & 0.61 & 1.8 \\
\hline Wagner Mix \#I & 0.135 & 1.69 & 2.9 & 0.060 & 0.75 & 3.3 \\
\hline
\end{tabular}

blobs retained; and $N_{\mathrm{b}}^{j}$ is the number of blobs retained. This equation expressing the volume of an effective sphere can then be solved for the effective diameter:

$$
d_{\mathrm{b}}^{j}=2\left(\frac{3 m_{\mathrm{b}}^{j}}{4 \pi \rho_{\text {sty }} N_{\mathrm{b}}^{j}}\right)^{1 / 3}
$$

The use of the liquid styrene density in these equations reduces the error associated with measuring dimensions of the "shrunken" polystyrene blobs. Effective diameters for multi-pore-space blobs which incorporate sand grains in their structure were corrected by multiplying the blob volume (right-hand side of Eq. 6) by the porosity. Statistics for the computed effective diameters are summarized in Table 5. Here, the median effective diameter $\left(d_{50_{\mathrm{b}}}\right)$ and uniformity index $\left(U_{\mathrm{i}_{\mathrm{b}}}=d_{60_{\mathrm{b}}} / d_{1 \mathrm{O}_{\mathrm{b}}}\right)$ based on blob number and mass distribution data are presented for each sand. Uniformity indices calculated from the number distributions are not reliable since, in several cases, $>10 \%$ of the total number of blobs were retained on the smallest sieve size employed to separate blobs. Therefore, it was difficult to quantify the fraction of blobs with effective diameters less than $d_{10_{b}}$. The ratios of median effective sphere size and median sand grain size $\left(d_{50_{\mathrm{g}}}\right)$ are also presented in this table to iiiustrate the relative size of blobs with respect to the sand grain size. The following observations can be made based on the calculated effective sphere diameters:

(1) The median effective sphere diameter, $d_{50_{b}}$, increases (Wagner $\# 50<$ Ottawa < Wagner \#18), with increasing grain size for uniform sands. These results support results of the recent work of Mayer and Miller (1992).

(2) A comparison of blobs entrapped in sands with similar median grain sizes (Ottawa and Wagner Mix \#l) indicates that the median effective diameter and the uniformity index of the blobs, as calculated from the mass distributions, increase with increasing gradation of sand grain sizes (Ottawa < Wagner Mix \#l). However, since the number of larger blobs entrapped in the graded medium is insignificant with respect to the total number of blobs entrapped, the median effective diameter and uniformity index do not exhibit significant differences when based on blob number distributions.

(3) Ratios of $d_{50_{\mathrm{b}}} / d_{50_{\mathrm{B}}}$ based on the mass fraction distribution are $\geqslant 1.0$, indicating 

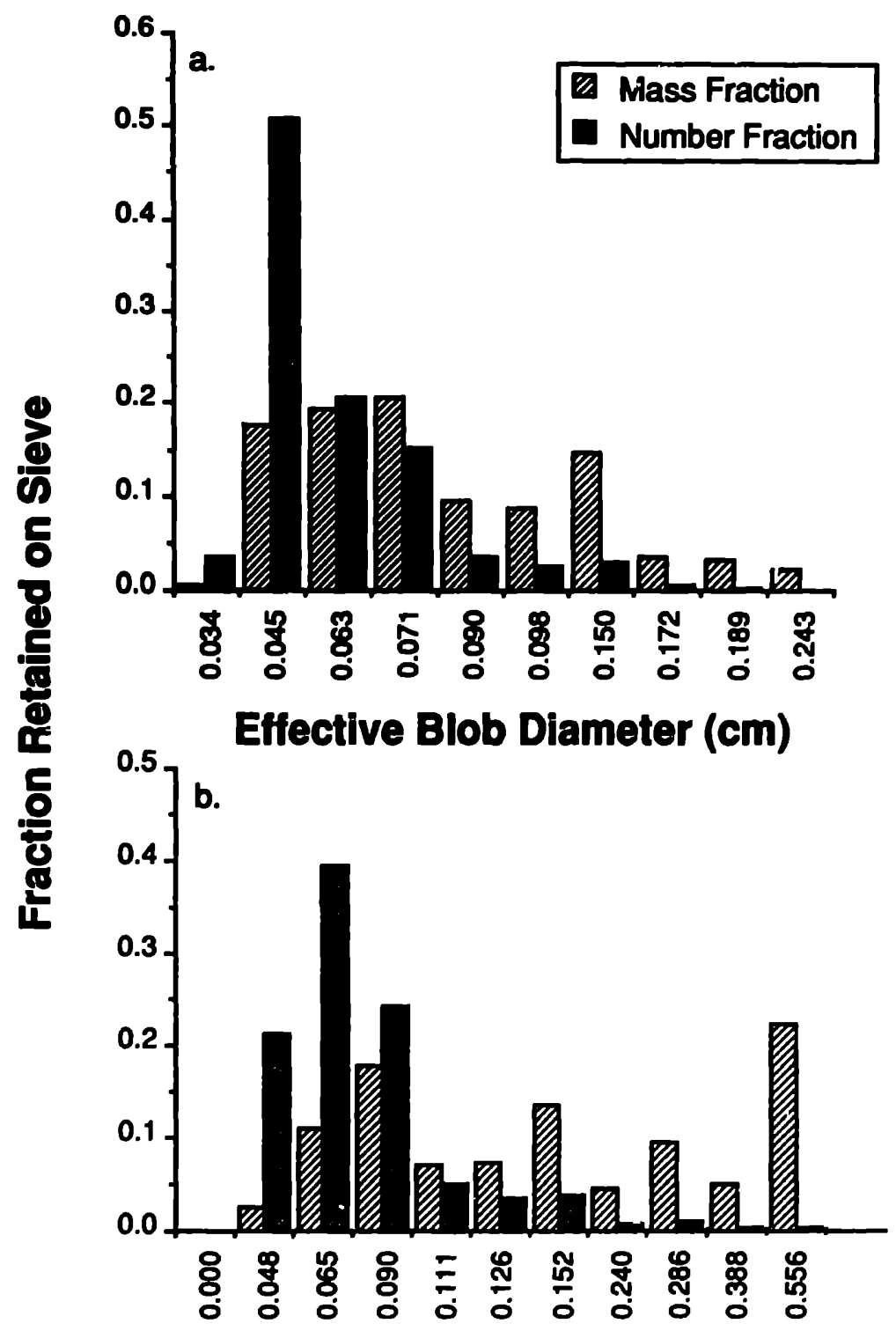

\section{Effective Blob Diameter (cm)}

Fig. 3. NAPL blob distributions from styrene polymerization and characterization analyses for: (a) uniform Wagner \#50 sand; and (b) graded Wagner Mix \#I sand.

that most of the mass is entrapped in blobs which are larger than the sand grain size in all sands.

(4) Ratios of $d_{50_{\mathrm{b}}} / d_{50_{\mathrm{g}}}$ based on the number fraction distribution are $<1.0$ for all sands tested, indicating that the greatest number of blobs are smaller than the median grain size.

From the above observations it can be inferred that, although most of the NAPL blobs exist as singlets in each water-wet sand, the distribution of larger blobs is highly dependent on porous medium type. Comparison of the largest blobs retained in the Ottawa vs. Wagner Mix \#I shows that the graded sand retains much larger and more amorphous blobs than a uniform sand of comparable median grain size. 
Powers et al. $(1991,1992)$ have illustrated that NAPL dissolution rates are very sensitive to the distribution of blob sizes within a porous medium. The data presented herein facilitate a more thorough analysis of the effect of blob distribution on dissolution rate and the development of a more "fundamental" mass-transfer model than those "lumped-domain" models utilized by Miller et al. (1990), Imhoff et al. (1994), and Powers et al. (1994).

\section{NAPL dissolution model}

The experimental results presented above were utilized in the development of a mathematical model describing the transient NAPL dissolution phenomenon occurring as pure-phase NAPL blobs dissolve and shrink over time. The basis of this model is the hypothesis that NAPL dissolution can be modeled with a linear-driving-force mass-transfer model (Eq. 1) by incorporating independently defined mass-transfer coefficients and specific surface areas. Mass-transfer coefficients were estimated from the correlation developed from naphthalene dissolution experiments (Eq. 5b), and the specific interfacial area was quantified by assuming that the NAPL blobs could be described by a set of spheres with a range of initial diameters. The data described by Powers et al. (1994) for the transient dissolution of styrene entrapped in one-dimensional columus were used to calibrate the model. Their transient TCE dissolution data sets were used to verify model predictions.

Critical to the development of this model, hereafter referred to as the "sphere model", is the incorporation of the transient nature of the dissolution process. During column flushing, NAPL blobs will shrink as mass is removed causing a reduction in interfacial area and a decrease in interstitial velocities as the effective porosity increases. To incorporate this variability of the interstitial velocity into the model, the mass-transfer expression which treats the Sherwood number as a function of Reynolds number based on pore-water velocity (Eq. 5b) was employed. The initial specific surface area $\left(a_{0}\right)$ was calculated from a distribution of effective blob diameters $\left(d_{\mathrm{b}}\right)$ :

$$
a_{\mathrm{o}}=\sum_{j=1}^{n} \frac{6 f_{\mathrm{r}}^{j} \theta_{\mathrm{n}}}{\epsilon d_{\mathrm{b}}^{j}} F^{j}
$$

where $\boldsymbol{n}$ is the number of size fractions considered; $f_{r}^{j}$ is the mass fraction retained on sieve $j$; and $\boldsymbol{F}^{j}$ is a parameter to describe discrepancies between actual blob shape and the idealized sphere geometry and to account for the fact that only a fraction of surface area is exposed to mobile water. The porosity term in the denominator accounts for the incorporation of sand grains in the total volume of large, amorphous blobs. Porosity was excluded from this expression for blobs expected to occupy one or two pore bodies.

The mass-transfer coefficient correlation (5b) and expression for specific surface area (8) were incorporated into a numerical simulator, GANGLIA, which was developed 
to solve the solute transport equation with a linear-driving-force mass-transfer term (Eq. 1) describing NAPL dissolution (Powers et al., 1991):

$$
\theta_{\mathrm{w}} \frac{\partial C}{\partial t}=\frac{\partial}{\partial x}\left(\theta_{\mathrm{w}} D_{\mathrm{h}} \frac{\partial C}{\partial x}\right)-q \frac{\partial C}{\partial x}+k_{\mathrm{f}} a_{\mathrm{o}}\left(C_{\mathrm{s}}-C\right)
$$

where $\theta_{\mathrm{w}}$ is the aqueous-phase volumetric fraction; $q$ is the superficial velocity; and $D_{\mathrm{h}}$ is the hydrodynamic dispersion coefficient. This equation can be rewritten in a dimensionless form to show the relationship with variables in the Sherwood number correlations:

$$
\theta_{\mathrm{w}} \frac{\partial \chi}{\partial \tau}=\frac{\partial}{\partial \xi}\left(\theta_{\mathrm{w}} \frac{1}{\operatorname{Pe}} \frac{\partial \chi}{\partial \xi}\right) \frac{\partial \chi}{\partial \xi}+\frac{\mathrm{Sh}}{\operatorname{Re} \cdot \mathrm{Sc}} \alpha(1-\chi)
$$

where the independent variables are given as $\xi=x / L$ and $\tau=q t / L$; the dependent variable becomes $\chi=C / C_{s}$; and the additional cocfficients are the Péclet number $\left(\mathrm{Pe}=q L / D_{\mathrm{h}}\right)$ and dimensionless surface area $\left(\alpha=a_{\mathrm{o}} L\right)$.

In GANGLIA, this quasi-linear partial differential equation (9) is solved with a Galerkin finite-element method discretization of the spatial derivatives and a backward finite-difference approximation of the time derivative. NAPL saturations are recomputed after each time step, using a mass-balance equation for the organic phase. The other transient parameters, $D_{\mathrm{h}}, \theta_{\mathrm{w}}, a_{\mathrm{o}}$ and $k_{\mathrm{f}}$, are then computed with the updated NAPL saturations. The evaluation of these parameters is lagged one time step behind the computation of nodal concentrations. Detailed equations describing these calculations have been presented previously (Powers et al., 1991).

\subsection{Model application and calibration}

To evaluate the applicability of the model, the numerical code, GANGLIA, was used to simulate column effluent concentrations for a series of transient styrene dissolution experiments (Powers, 1992; Powers et al., 1994). For these simulations, mass fractions and effective blob diameters for each size fraction (as shown in Fig. 3) were used to calculate the initial specific surface area (Eq. 8). The parameter $F^{j}$ appearing in Eq. 8 was used to calibrate model predictions to the experimental data. Recall that this parameter represents the fraction of surface area exposed to mobile water and accounts for discrepancies between actual blob geometries and idealized sphere geometries.

$F$-values were determined by the adjustment of this parameter to fit numerical simulations to experimental data. The best fit for the $F$-values was achieved with an iterative approach which employed the method of least squares to quantify the error in the logarithm of normalized concentrations between experimental data (expt) and model simulations (sim):

$$
\text { (error) }=\frac{1}{n} \sum_{i=1}^{n}\left[\log \left(C / C_{\mathrm{s}}\right)_{\mathrm{expt}}^{i}-\log \left(C / C_{\mathrm{s}}\right)_{\text {sim }}^{i}\right]^{2}
$$

Here $n$ is the number of experimental data points. The logarithm of concentrations was employed so that discrepancies in concentrations would be weighted equally over 


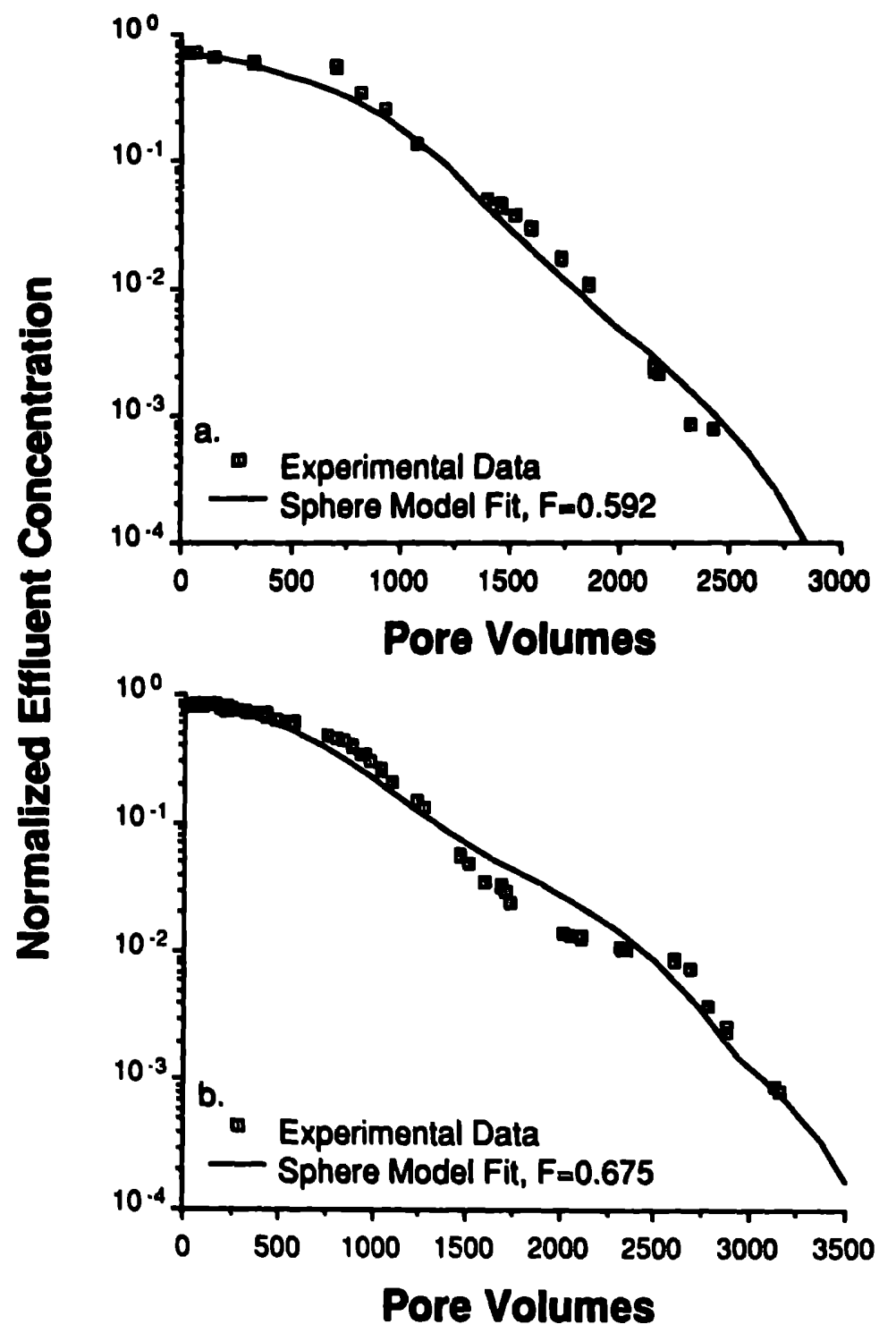

Fig. 4. Best-fit sphere model simulations of transient styrene dissolution data: (a) uniform Ottawa sand $\left(q=9.1 \mathrm{~m} \mathrm{day}^{-1} ; \theta_{\mathrm{n}}=0.049\right)$; and (b) graded Wagner Mix \# $l$ sand $\left(q=8.2 \mathrm{~m} \mathrm{day}^{-1} ; \theta_{\mathrm{n}}=0.065\right)$.

the three or four order of magnitude range in effluent concentrations. The value of $F$ for each experimental data set was determined by selecting an initial estimate of this coefficient, running the GANGLIA code to simulate the effluent concentrations over time, and then calculating the differences between numerical simulations and experimental data. This procedure was repeated in an iterative fashion until an error minimum was determined to within a tolerance of $F \pm 0.005$. Values of $F$ and corresponding errors (Eq. 11) for each of the transient experimental data sets are summarized in Table 6. To minimize the number of fitting parameters used in this model, it was assumed that one value of $F$ would be appropriate for all NAPL blob size fractions.

Statistical analysis of values of the best-fit $F$ parameters for each of the transient styrene dissolution experiments indicated that, with the limited data available, there is 
Table 6

Values of $\boldsymbol{F}$ for sphere model

\begin{tabular}{llll}
\hline Sand & $\begin{array}{l}\text { Aqueous-phase velocity } \\
\left(\mathrm{m} \mathrm{day}^{-1}\right)\end{array}$ & $F$ fit to data sets & Error \\
\cline { 2 - 5 } & 3.5 & 0.63 & \pm 0.0073 \\
Wagner \#50 & 3.8 & 0.60 & \pm 0.0161 \\
Ottawa & 9.1 & 0.592 & \pm 0.0014 \\
Ottawa & & $(0.596)^{\mathrm{b}}$ & \\
Ottawa & 3.8 & 0.48 & \pm 0.0062 \\
Wagner \#18 & 3.7 & 0.94 & \pm 0.0242 \\
Wagner Mix \#I & 8.2 & 0.675 & \pm 0.0135 \\
Wagner Mix \#I & & $(0.81)^{\mathrm{b}}$ & \\
Wagner Mix \#I & & & \\
\hline
\end{tabular}

aDefined by Eq. 11.

${ }^{b}$ Average $F$-value for each sand in parentheses.

no significant correlation between this parameter and grain size, uniformity index or aqueous-phase velocity at an $\alpha=0.01$ level of statistical significance.

In their work, Mayer and Miller (1992) employed a shape factor, $\psi$, to describe the difference in the volume of polymerized styrene blob volumes to the volume of a sphere with an equivalent effective length. Although this definition is slightly different from that of the $F$ factor used herein to describe discrepancies between actual blob geometry and ideal spherical shape, the two parameters can be compared. As observed in this study, Mayur and Miller (1992) found little dependence of the shape factor on the median grain diameter. They confirm, however, that the shape factor changes with blob size within a uniform porous medium. Small blobs have a shape factor equal to 1 , indicating that they are spherical, while the shape factor of larger spheres decreases with increasing size to a minimum of $\psi \approx 0.25$. The range of calibrated $F$-values determined in this study $(F=0.48-0.94)$ is consistent with the analysis of Mayer and Miller (1992).

Fig. $4 a$ and $b$ illustrates simulations of effluent concentrations from two of the styrene dissolution column experiments (Powers, 1992; Powers et al., 1994). Here, the effluent solute concentrations are plotted as normalized concentrations $\left(C / C_{\mathrm{s}}\right)$ and the "time" scale is represented as cumulative pore volumes of water pumped through the column. To avoid the complications of the transient water-filled pore volume, pore volumes shown in Fig. 4 are defined in terms of the total void volume of the column. The $F$-values determined through calibration (Table 6) were used to generate these simulatioris. Sphere model simulations were able to describe trends in data over the period of time required to remove NAPL from column systems. Column effluent data from graded sands (Fig. 4b) exhibited characteristic curvature changes which may be interpreted as reflecting changes over time in the blob size dominating dissolution. Note that the sphere model also predicts these changes in slope as small blobs disappear from the system. The overall trend of the sphere model simulations are consistent with the data. However, the shapes of the predicted effluent concentration curves do not 


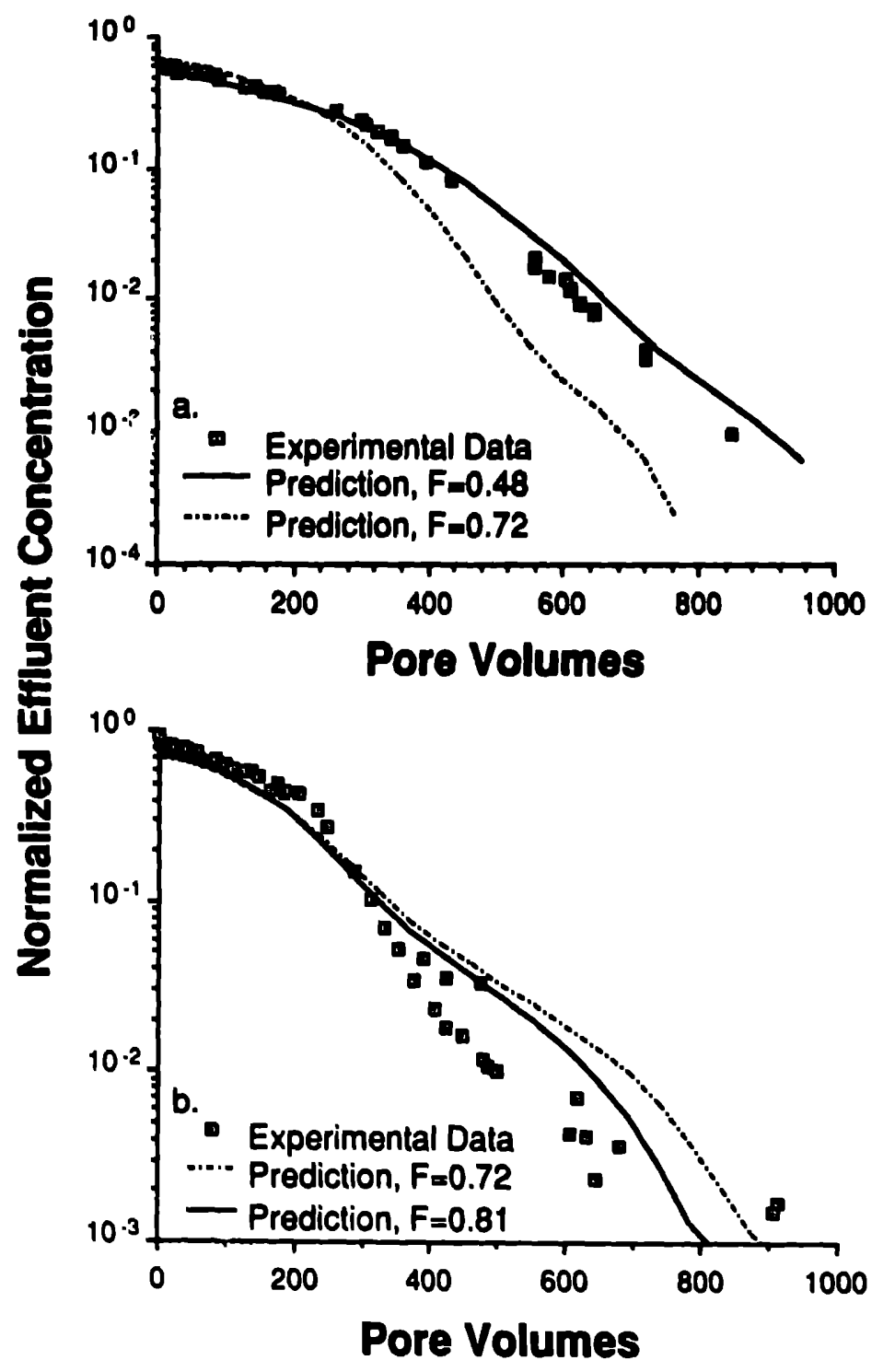

Fig. 5. Sphere model predictions of transient TCE dissolution data: (a) uniform Wagner \#/8 sand $\left(q=3.8 \mathrm{~m} \mathrm{day}^{-1} ; \theta_{\mathrm{n}}=0.050\right)$; and (b) graded Wagner Mix \#I sand $\left(q=7.4 \mathrm{~m} \mathrm{day}^{-1} ; \theta_{\mathrm{n}}=0.050\right)$.

precisely mimic those of the measured values. Using a range of $F$-values for different sphere sizes or changing the proportion of NAPL volume associated with each size fraction improved the model's ability to match the data. These changes were very subjective, however, and could not be justified with the limited data available.

\section{j.2. Model verification}

To explore the predictive capacity of the sphere model for similar column systems, average values of $F$ obtained from the styrene dissolution calibration were used to 
predict effluent concentrations for TCE dissolution experiments (Powers, 1992; Powers et al., !994). Two different averages of $F$-values were considered for the prediction of transient TCE dissolution data sets: an average value of $F$ from calibration of all styrene dissolution experiments $(F=0.72)$ and an average value of $F$ within each sand type (Table 6). Since mass and size distributions of effective spherical blobs are also required as input parameters, this model is only capable of predicting NAPL dissolution in sands for which a reasonable estimate of the initial NAPL distribution is available.

Comparison of model predictions to experimental data is illustrated in Fig. 5a and b. Using the calibrated $F$-value for the Wagner $\# 18$ styrene dissolution data set, the model was an excellent predictor of TCE dissolution from the coarse, uniform Wagner \#18 sand (Fig. 5a). Powers (1992) shows that the model was also an excellent predictor of entrapped TCE dissolution from Ottawa sand. Prediction of effluent concentrations from experiments utilizing a graded sand (Wagner Mix \#I) (Fig. 5b) were not as good as predictions of dissolution from the uniform sands. The sphere model did not adequately reproduce the changes in slope of the data at appropriate points. In all cases, sphere model predictions based on average $F$-values for each sand were superior to those based on the overall average $F$-value $(F=0.72)$.

In the present application, it was assumed that the NAPL blob distributions measured for styrene entrapped and polymerized in one column experiment would be appropriate for all experiments involving NAPL entrapped in that same medium. Observed discrepancies among calibrated $F$-values for a particular medium and between model predictions and experimental data suggest that there could be differences in the actual NAPL blob distributions. Mayer and Miller (1992) have shown that the required size of a representative elemental volume (REV) for multiphase processes is greater than that for other porous r.tedium characteristics, such as porosity. With the use of polystyrene distribution data, their analyses show that, in order to quantify residual saturations of NAPL entrapped in uniform porous media to within $\pm 1 \%$ of the true value, REV's on the order of $10-300 \mathrm{~cm}^{3}$, depending on the grain size, are required. They extrapolate their findings to predict that even larger REV's would be required for porous media that are less uniform than the glass beads they employed. The column volumes that were used in the present study for averaging residual saturations and NAPL blob distributions ranged from 75 to $100 \mathrm{~cm}^{3}$. Consistency in the $F$-values determined for dissolution from Ottawa sand and the quality of the predicted effluent concentrations for TCE dissolution from the Wagner \#I8 sand suggest that the NAPL blob distributions determined for those sands were probably representative. For the graded Wagner Mix \#I sand, however, there were greater discrepancies among the calibrated $F$-values determined from separate experiments (Table 6) and greater error between model predictions and TCE effluent data (Fig. 5b). Based upon the above discussion, these di Terences may be attributed, in part, to variations in NAPL blob distributions entrapped in this sand among the various experiments. Perhaps, larger column volumes, which are more closely representative of the appropriate REV size, would provide more accurate blob size distribution data for this graded medium. 


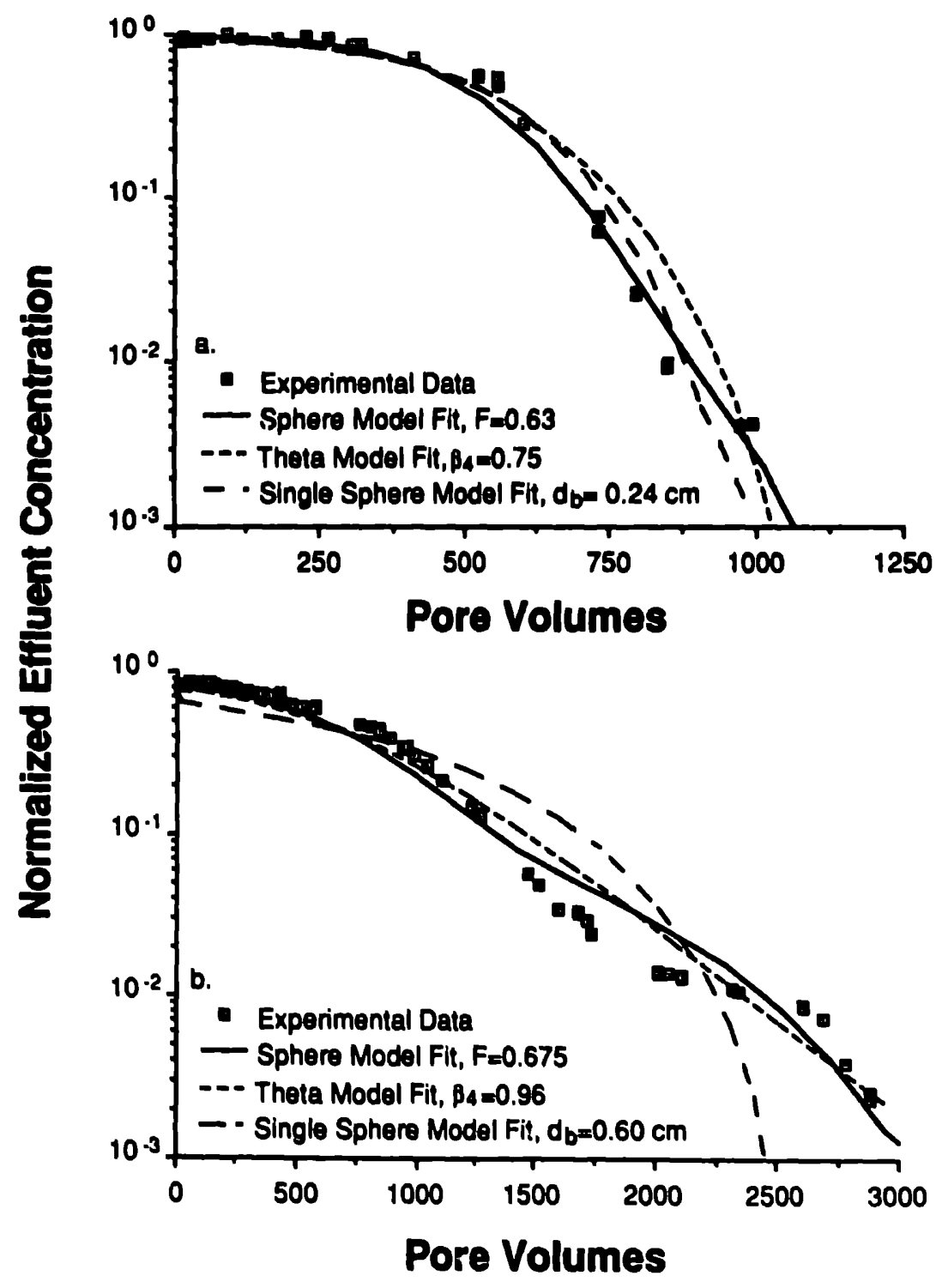

Fig. 6. A comparison of simulations from several different phenomenological models for styrene dissolution: (a) uniform Wagner \#50 sand $\left(q=3.5 \mathrm{~m}^{-1}\right.$ day $\left.^{-1} ; \theta_{\mathrm{n}}=0.045\right)$; and (b) graded Wagner Mix \#I sand $\left(q=8.2 \mathrm{~m} \mathrm{day}^{-1} ; \theta_{\mathrm{n}}=0.065\right)$.

\section{Discussion}

The transient NAPL dissolution model developed herein has been shown to adequately simulate transient NAPL dissolution data for a range of sand types. As discussed previously, three alternative phenomenological models have also been utilized in the literature for transient entrapped NAPL blob-water mass-transfer processes: lumped-domain models (Miller et al., 1990; Imhoff et al., 1994; Powers et al., 1994); single-sphere-size models (Geller and Hunt, 1993); and two-site models (Robinson and Bedient, 1991; Borden and Kao, 1992; Brusseau, 1992). The complex two-site model, however, may not be warranted for the sands considered herein. The conclusions of Hatfield et al. (1993) suggest that, in medium-coarse sandy soils, the 
NAPL-mobile water dissolution rates represent the primary rate-limiting phenomenon. Therefore, for these experimental data, it is probable tha! with the two-site model, any improvement in the capability of this model to fit data would be the result of multiple fitting parameters, not an improvement in the conceptual model of phenomena.

A comparison of the other models for NAPL-water interphase mass transfer can provide insight into the NAPL dissolution process. This evaluation can be made on several levels, including: ability to fit experimental data; ability to predict experimental data; and availability of accurate input parameters. The numerical simulator, GANGLIA, was modified to incorporate these alternate models so that simulations of effluent concentrations from one-dimensional column systems could be coinpared. Model simulations were compared to data from the dissolution of en!rapped styrene in the uniform Wagner \#50 and the graded Wagner Mix \#l sands (Fig. 6).

\subsection{Single-sphere models}

The single-sphere size model used by Geller and Hunt (1993) was developed for NAPL dissolution in a porous medium comprised of uniform size glass beads. It is likely that NAPL blobs entrapped in such a medium may be well described by a uniform sphere size. Simulations presented in Fig. 6, however, demonstrate that in more a complex medium, this model does not adequately describe effluent data. The single-sphere size model adequately represents effluent concentrations from a medium-fine uniform sand (Fig. 6a) due to the relatively narrow range of blob sizes retained in this medium (Fig. 3a). The single-sphere size model is not, however, representative of effluent concentrations from NAPL dissolution in a graded medium. The presence of a few large blobs in the graded medium (Fig. 3b) creates the S-shaped data curve (Fig. 6b) which is not mimicked by tine single-sphere model. It is anticipated that these discrepancies between the predictions of the single-sphere model and actual aqueous-phase concentrations would be magnified in a more heterogeneous soil system.

The single-sphere size model simulations presented in Fig. 6 were calibrated in the inanner described by Geller and Hunt (1993). That is, the $F$ parameter was assumed to equal 1.0 and the initial sphere diameter was utilized as a calibration parameter. The calibrated effective blob diameters determined for this model (Wagner \#50: $d_{\mathrm{b}}=0.24$ $\mathrm{cm}$; Wagner Mix \#I: $d_{\mathrm{b}}=0.60 \mathrm{~cm}$ ), are significantly higher than the median diameters determined through the polystyrene blob size analysis (Wagner \#50: $d_{50_{\mathrm{b}}}=0.07 \mathrm{~cm}$; Wagner Mix $\# 1: d_{50_{\mathrm{b}}}=0.14 \mathrm{~cm}$ ). This may indicate that the larger blob sizes dominate the overall mass-transfer process. The ratios of calibrated "effective" sphere size to median sand grain size for the Wagner \#50 $\left(d_{\mathrm{b}} / d_{50_{\mathrm{g}}}=5.3\right)$ and the Wagner Mix $\# l$ sand $\left(d_{\mathrm{b}} / d_{50_{\mathrm{g}}}=7.5\right)$ were both within the range of 3.5-10 found by Geller and Hunt (1993).

Presently, data are insufficient for appiication of the single-sphere model to predict effluent concentrations from column experiments. Geller (1990) showed that this model is fairly sensitive to the value of the initial sphere diameter over a reasonable range of estimates, but not as sensitive to the estimated residual saturation. Sensitivity 
to sphere diameter was also illustrated by Powers et al. (1991). A means of accuraisly estimating this parameter is required in order to use the single-sphere model in a predictive mode.

\subsection{Lumped-domain models}

Lumped-domain models treat the dissolving NAPL as a "black box", that is, no knowledge of the actual distribution of NAPL blob size is required. These models employ lumped mass-transfer coefficients $\left(\hat{k}=k_{\mathrm{r}} a_{\mathrm{o}}\right)$ and account for the reduction in mass-transfer rates over time by correlating modified Sherwood numbers to the volumetric fraction of NAPL.

Powers et al. (1994) discuss the ability of their lumped-domain ("theta") model to fit the same transient styrene dissolution data employed in the present work. This model utilizes a correlation for a modified Sherwood number as a function of the system hydrodynamics, pore structure and volumetric fraction of NAPL:

$$
\mathrm{Sh}^{\prime}=\frac{\hat{k} d_{50}^{2}}{D_{\mathrm{L}}}=4.13 \mathrm{Re}^{0.598} \delta_{\mathrm{g}}^{0.673} U_{\mathrm{i}_{\mathrm{g}}}^{0.369}\left(\frac{\theta_{\mathrm{n}}}{\theta_{\mathrm{1}_{\mathrm{o}}}}\right)^{\beta_{4}}
$$

where $\theta_{\mathrm{n}_{\mathrm{o}}}$ is the initial volumetric fraction of NAPL in the system. This correlation was employed to estimate lumped mass-transfer coefficients at each time step and nodal location within the GANGLIA simulator. The effect of porous medium properties on the size distribution of the entrapped NAPL is incorporated through the inclusion of the normalized median grain diameter and the uniformity index. Differences in the way the specific surface area change as blobs dissolve are incorporated by allowing the $\beta_{4}$ coefficient to vary among media. Optimal values of this coefficient were assessed in a manner analogous to the sphere modei calibration discussed above. A correlation equation was presented to estimate the $\beta_{4}$ calibration parameter in terms of the median grain size and uniformity index of the medium. Use of this correlation allows the theta model to be applied to similar sandy soils with known grain-size distributions.

Examples of the quality of fit of the theta model to transient styrene dissolution data sets are presented in Fig. 6. Model simulations match overall trends in the data reasonably well, but the predicted concentration distributions were consistently more concave than experimental data from medium-fine uniform sands (Fig. 6a). However, a comparison of errors (Eq. 11) between model simulations and experimental data calculated for the sphere and theta models indicated that these models had comparable abilities to fit transient styrene dissolution data (Powers, 1992).

The theta model (Püwers et al., 1994) requires grain-size distribution parameters for the sandy media and an estimate of initial residual saturation throughout the domain. Grain-size parameters are easy to obtain from sieve analyses. The initial residual saturation is more difficult to accurately estimate. Powers et al. (1994) illustrate that aqueous-phase effluent concentration profiles predicted by the theta model are highly sensitive to this parameter.

The lumped-domain models presented by Miller et al. (1990) and Imhoff at al. 
(1994) were developed for a limited range of porous medium characteristics and do not contain calibration parameters that allow them to be extrapolated to other similar media. This limits their use to porous media similar to those used for their development.

\subsection{Sphere model}

The results of the present work illustrate the applicability of the sphere model to the dissolvtion of NAPL's entrapped in sandy media. The potential limitation of this type of model, however, is the input data required. These data requirements are much greater for the sphere model than for the theta or single-sphere models. A distribution of effective sphere diameters representative of the actual NAPL blob sizes is required. St! rene polymerization and blob characterization were conducted as part of this research work to define these parameters. While this procedure was effective for the sands considered, it is a very time-intensive procedure which reduces its feasibility as a method for parameter estimation. Thus, to support sphere model applications, additional research would have to be undertaken to develop methods for estimating a distribution of effective sphere diameters from easily measurable soil properties.

The predictive capability of the sphere model is also limited by the need for accurate estimates of the $F$ calibration parameter. For this work, it was determined that calibration of this parameter with transient styrene dissolution data from the same porous medium was an effective means of estimating the appropriate value of $F$ for prediction of TCE dissolution data. However, no method was developed to estimate $\boldsymbol{F}$ for porous media other than those tested in this research.

While data requirements for the sphere model may seem onerous, the independent assessment of mass-transfer coefficients and specific surface areas makes it more versatile than the other available models. The theta model, with its present procedure for estimating the calibration parameter (Powers et al., 1994) is limited to prediction of NAPL dissolution from laboratory scale, homogeneous sand columns. The calibrated model would not be appropriate for heterogeneous systems. The sphere model could, however, be extrapolated to heterogeneous systems if future research efforts lead to a better understanding of the pore-scale distribution of NAPL within heterogeneous porous media. The sphere modeling approach may also prove useful in the simulation of other remediation technologies, such as surfactant flushing. This would require the development of a mass-transfer coefficient correlation applicable to surfaciant systems.

\section{Conclusions}

A phenomenological model for transient NAPL dissolution was devcloped. In this model, the mass-transfer coefficient and specific surface areas are quantified 
independently. It is unique due to the assumption that the complex distribution of NAPL blob shapes and sizes can be represented by a set of spheres with a range of diameters.

A correlation for mass-transfer coefficients was generated from naphthalene sphere dissolution data. This correlation was compared with a number of correlations that could be considered analogous to the NAPL dissolution system. Due to the large discrepancies among estimated mass-transfer coefficients, it was concluded that it is important to use a consistent definition of the mass-transfer coefficient and to employ correlations which were developed for systems with similar conditions to those of interest. The trend of high values of the $\beta_{1}$ coefficient for naphthalene sphere and NAPL dissolution correlations at low Reynolds numbers suggests that the pore-scale hydrodynamics of these systems are similar, and that they differ from those in packed beds of large, uniform size spheres at low Reynolds numbers. It may be concluded from these trends that aquenus-phase velocities near the NAPL- water interface in porous media are probably higher than at comparable Reynolds numbers in beds packed with large spheres.

Polystyrene blob distributions were used to estimate the NAPL-water specific surface area. Effective sphere diameters for each set of blobs were defined so that the sphere volume would equal the average blob volume. The distribution of effective diameters showed the same trends in relative size and size distribution as did the less quantitative comparison of blob sizes presented by Powers et al. (1992).

Numerical simulations of effluent concentrations from transient styrene dissolution experiments previously presented by Powers et al. (1994) showed that the sphere model can be calibrated to experimental data. The inclusion of a range of sphere sizes resulted in a model which represents experimental data better than a model based on mono-size spheres. The significance of these differences was related to the type of porous medium considered; the single-sphere size model fit data from medium-fine sand adequately, but did not represent trends in effluent concentration data from a graded sand. This can be explained by the distribution of blob sizes in each of these media. The phenomenological model developed in the present work was capable of describing NAPL dissolution processes from media in which a wide range of blob sizes was entrapped.

Given enough data for a system, the sphere model adequately predicts dissolution for NAPL's with a range of solubilities. This, however, requires quantification of the calibration factor, $F$, for the porous medium of interest. With the limited data available in this work, the only method of estimating this parameter was by calibrating to existing dissolution data from the sand of interest.

The primary limitation of the sphere model is the quantity of input data required to describe the distribution of effective sphere sizes. The styrene blob polymerization, separation and characterization methods employed herein are tedious and are not considered a feasible method of parameter estimation for more than a few samples. While these data requirements are extensive, the ability to define the NAPL blob distribution within the model makes the sphere model much more versatile for extrapolation to heterogeneous systems than any of the other available dissolution models. 


\section{Acknowledgements}

Funding for this research was provided in part by the U.S. Department of Energy, Office of Health and Environmental Research, Subsurínce Science Program (Contract \# DE-F60289-ER60844) under the project direction of Dr. Frank Wobber, and in part by the U.S. Public Health Service Grant ES04911 from the National Institute of Environmental Health Sciences.

\section{References}

Abriola, L.M., 1989. Modeling migration of organic chemicals in groundwater systems: $A$ review and assessment. Environ. Health Persp., 83: 117-143.

Anderson, M.R., Johnson, R.L. and Pankow, J.F., 1992. Dissolution of dense chlorinated solvents into groundwater, 1. Dissolution from a well defined residual source. Ground Water, 30: 250-256.

Borden, R.C. and Kao, C.M., 1992. Evaluation of groundwater extraction for remediation of petroleum contaminated groundwater. Water Environ. Res., 64: 28-36.

Boundy, R.H., Boyer, R.F. and Stoesser, S.M., 1965. Styrene: Its Polymers, Copolymers and Derivatives, Part I. Hafner, New York, NY, 212 pp.

Bowman, C.W., Ward, D.M., Johnson, A.I. and Trass, O., 1961. Mass transfer from fluid and solid spheres at low Reynolds numbers. Can. J. Chem. Eng., 39: 9-13.

Brusseau. M.L., 1992. Rate-limited mass transfer and transport of organic solutes in porous media that contain immobile immiscible organic liquid. Water Resour. Res., 28: 33-46.

Chatzis, I., Morrow, N.R. and Lim, H.T., 1983. Magnitude and detailed structure of residual oil saturation. Soc. Pet. Eng. J., 24: 311-326.

Cherry, J.A., Feenstra, S., Kueper, B.H. and McWhorter, D.W., 1990. Status of in-situ technologies for cleanup of aquifers contaminated by DNAPLs below the water table. Presented at Int. Spec. Conf. on How Clean is Clean? - Cleanup Criteria for Contaminated Soil and Groundwater. Air Waste Manage. Assoc., Washinglon, DC, Nov. 6-9, 1990.

Cohen, R.M., Mercer, J.W. and Matthews, J., IS43 DNAPL site investigation. C.K. Smoley, Inc., Boca Raton, FL, 339 pp.

Conrad, S.H., Wilson, J.L., Mason, W.R. and Peplinski, W.J., 1992. Visualization of residual organic liquid trapped in aqu:fers. Water Resour. Res., 28: 467-478.

deZabala, E.F. and Radke, C.J., 1986. A non-equilibrium description of alkaline water flooding. Soc. Pet. Eng. J., 26: 29-43.

Driscoll, F.G., 1986. Groundwater and Weils. Johnsun Filtration Systems, Inc., St. Paul, MN, 2nd ed., $1089 \mathrm{pp}$.

Dwivedi, P.N. and Upadhyay, S.N., 1977. Particle - fluid mass transfer in fixed and fluidized beds. Ind. Eng. Cheill. Fiucess Des. Dev., i6: 157-165.

Fedkiw, P. and Newman, J., 1978. Low Peclet number behavior of the transfer rate in packed beds. Chem. Eng. Sci., 33: 1043-1048.

Feenstra, S. and Coburn, J., 1986. Subsurface contamination from spills of denser than water chlorinated solvents. Calif. Water Pollut. Control Assoc. Bull., 23: 26-34.

Fountain, J.C., Klimek, A., Beikirch, M.G. and Middleton, T.M., 1991. The use of surfactants for in situ extraction of organic pollutants from a contaminated aquifer. J. Hazard. Mater., 28: 295-311.

Frie Jlander, S.K., 1957. Mass and heat transfer to single spheres and cylinders at low Reynolds numbers. AIChE (Am. Inst. Chem. Eng.) J., 3: 43-48.

Geller, J.T., 1990. Dissolution of non-aqueous phase organic liquids in porous media. Ph.D. Dissertation, University of California at Berkeley, Berkeley, CA, 184 pp.

Geller, J.T. and Hun', I.R., 1493. Mass transfer from innaqueous phase organic liquids in water-saturated porous media. Water Resour. Res., 29: 833-846. 
Hatfield, K., Ziegler, J. and Burris, D.R., 1993. Transport in porous media containing residual hydrocarbon, II: Experiments. J. Environ. Eng., 119: 559-575.

Hunt, J.R., Sitar, N. and Udell, K.S., 1988. Non-aqueous phase liquid transport and cleanup, 1. Analysis of mechanisms. Water Resour. Res., 24: 1247-1258.

Imhoff, P.T., Miller, C.T. and Thyrum, G., 1993. An analysis and experimental study of dissolution fingers. Eos (Trans. Am. Geophys. Union), 74: 149 (abstract).

Imhoff, P.T., Jaffe, P.R. and Pinder, G.F., 1994. An experimental study of complete dissolution of nonaqueous phase liquid in saturated porous media. Water Resour. Res., 30: 307-320.

Johnson, R.L. and Pankow, J.F., 1992. Dissolution of dense chlorinated solvents into groundwater, 2. Source functions for pools of solvent. Environ. Sci. Technol., 26: 896-901.

Keely, J.F., 1989. Performance evaluations of pump-and-treat remediations. U.S. Environ. Prot. Agency, Off. Res. Dev., Ofr. Solid Waste Eniergency Response, Washington, DC, EPA/504/4-89-005, 86 pp.

Kumar, S., Upadhyay S.N. an I Mathur, V.K., 1977. Low Reynolds number mass transfer in packed beds of cylindrical particles. Ind. Eng. Chem. Process Des. Dev., 16: 1-8.

Larson, R.G., Davis, H.T. and Scriven, L.E., 1981. Displacement of residual nonwetting fluid from porous media. Chem. Eng. Sci., 36: 75-85.

Mackay, D.M. and Cherry, J.S., 1989. Groundwater contamination: Pump-and-treat remediation. Environ. Sci. Technol., 19: 384-392.

Mayer, A.S. and Miller, C.T., 1992. The influences of porous medium characteristics and measurement scale on pore-scale distributions of residual nonaqueous phase liquids. J. Contam. Hydrol., 11: 189213.

Mayer, A.S. and Miller, C.T., 1993. An experimental investigation of pore-scale distributions of nonaqueous phase liquids at residual saturation. Trans. Porous Media, 10: 57-80.

Mercer, J.W. and Cohen, R.M., 1990. A review of immiscible fluids in the subsurface: Properties, models, characterization, and remediation. J. Contam. Hydrol., 6: 107-163.

Miller, C.T., Poirier-McNeill, M.M. and Mayer, A.S., 1990. Dissolution of trapped nonaqueous phase liquids: Mass transfer characteristics. Water Resour. Res., 26: 2783-2796.

Mohanty, K.K., Davis, H.T. and Scriven, L.E., 1987. Physics of oil entrapment in water wei rocks. Soc. Pet. Eng. Reservoir Eng., 2: 113-127.

Morrow, N.R., 1971. Small-scale paching heterogeneities in porous sedimentary rock. Bull. Assoc. Pet. Geol., 55: 514-522.

Morrow, N.R. and Chatzis, I., 1981. Measurement and correlacion of conditions for entrapment and mobilization of residual oil. U.S. Dep. Energy, Bartlesville, OK, Rep. DOE/BETC/3251-12, 110 pp.

Morrow, N.R., Chatzis, I. and Taber, J.J., 1988. Entrapment and mobilization of residual oil in bead packs. Soc. Pet. Eng. Reservoir Eng., 3: 211-218.

Nelson, P.A. and Galloway, T.R., 1975. Particle-to-fluid heat and mass transfer in dense systems of fine particles. Chem. Eng. Sci., 30: 1-6.

Ng. K.M., Davis, H.T. and Scriven, L.E., 1978. Visualization of blob mechanism in flow through porous media. Chem. Eng. Sci., 33: 1009-1017.

Pennell, K.D., Abriola, L.M. and Weber, Jr., W.J., 1993. Surfactant enhanced solubilization of residual dodecane in soil columns, 1. Experimental investigation. Environ. Sci. Technol., 27: 2332-2340.

Pfannkuch, H.O., 1984. Determi.lation of the contaminant source strength from mass exchange processes at the petroleum-groundwater interface in shallow aquifer systems. In: Petroleum Hydrocarbons and Organic Chemicals in Ground Water. Natl. Water Well Assoc., Worthington, OH, pp. 444-458.

Powers, S.E., 1992. Dissolution of nonaqueous phase liquids in saturated subsurface systems. Ph.D. Dissertation, The University of Michigan, Ann Arbor, MI, $180 \mathrm{pp}$.

Powers, S.E., Loureiro, C.O., Abriola, L.M. and Weber, Jr., W.J., 1991. Theoretical study of the significance of nonequilibrium dissolution of nonaqueous phase liquids in subsurface systems. Water Resour. Res., 27: 463-477.

Powers, S.E., Abriola, L.M. and Weber, Jr., W.J., 1992. An experimental investigation of NAPL dissolution in saturated subsurface systems: Steady-state mass transfer rates. Water Resour. Res., 28: 2691-2706. 
Powers, S.E., Abriola, L.M. and Weber, Jr., W.J., 1994. An experimental investigation of NAPL dissolution in saturated subsurface systems: Transient mass transfer rates. Water Resour. Res., 30: 321 332.

Ranz, W.E., 1952. Friction and transfer coefficients for single particles and packed beds. Chem. Eng. Progr., 48: 247-253.

Robinson, G.C. and Bedient, P.B., 1991. Modeliıg a time-variant source of contamination. In: Proceedings - Petroleum Hydrocarbons and Organic Chemicals in Groundwater: Prevention, Detection and Restoration. Natl. Water Well Assoc., Worthington, OH, pp. 531-540.

Schwille. F., 1988. Dense Chlorinated Solvents in Porous and Fractured Media. Lewis, Chelsea, MI, 146 pp. (translated by J.F. Pankow).

Treybal, R.E., 1980. Mass-Transfer Operations. McGraw-Hill, New York, NY, 3rd ed., 748 pp.

van Dam, J., 1967. The migration of hydrocarbons in a water bearing stratum. In: P. Hepple (Editor), The Joint Problems of the Oil and Water Industries. Inst. Pet., London, pp. 55-96.

Verschueren, K., 1983. Handbook of Environmental Data on Organic Chemicals. Van Nostrand Reinhold, New York, NY, 1310 pp.

Wakao, N. and Funazkri, T., 1978. Effect of fluid dispersion coefficients on particle-to-fluid mass transfer coefficients in packed beds. Chem. Eng. Sci., 33: 1375-1384.

Walpole, R.E. and Myers, R.H., 1989. Probability and Statistics for Engineers and Scientists. Macmillan, New York, NY, 4th ed., 765 pp.

Wardlaw, N.C., 1982. The effects of geometry, wettability, viscosity and interfacial tension on trapping in single pore-throat pairs J. Can. Pet. Technol., 21: 21-27.

Weber, Jr., W.J., 1972. Physicochemical Processes for Water Quality Control. Wiley, New York, NY, $640 \mathrm{pp}$.

Welty, J.R., Wicks, C.E. and Wilson, R.E., 1969. Fundamentals of Momentum, Heat and Mass Transfer. Wilcy, New York, NY, 697 pp.

Wilson, E.J. and Geankoplis, C.J., 1966. Liquid mass transfer at very low Reynol 's numbers in packed beds. Ind. Eng. Chem. Fund., 5: 9-14.

Wilson, J.L. and Conrad, S.H., 1988. Is physical displacement of residual hydrocarbons a realistic possibility in aquifer restoration? In: Proceedings - Petroleum Hydrocarbons and Organic Chemicals in the Subsurface: Prevention, Detection, Restoration. Natl. Well Water Assoc., Worthington, OH, pp. $107-133$. 